\title{
Near-IR echelle spectroscopy of Class I protostars: Mapping Forbidden Emission-Line (FEL) regions in [Fell]
}

\author{
C. J. Davis ${ }^{1}$, E. Whelan ${ }^{2}$, T. P. Ray ${ }^{2}$, and A. Chrysostomou ${ }^{3}$ \\ 1 Joint Astronomy Centre, 660 North A'ohōkū Place, University Park, Hilo, Hawaii 96720, USA \\ 2 Dublin Institute for Advanced Studies, School of Cosmic Physics, 5 Merrion Square, Dublin 2, Ireland \\ ${ }^{3}$ Department of Physical Sciences, University of Hertfordshire, Hatfield, Herts AL10 9AB, UK
}

Received 27 August 2002 / Accepted 22 October 2002

\begin{abstract}
Near-IR echelle spectra in [FeII] $1.644 \mu \mathrm{m}$ emission trace Forbidden Emission Line (FEL) regions towards seven Class I HH energy sources (SVS 13, B5-IRS1, IRAS 04239+2436, L1551-IRS5, HH34-IRS, HH 72-IRS and HH 379-IRS) and three classical T Tauri stars (AS 353A, DG Tau and RW Aur). The parameters of these FEL regions are compared to the characteristics of the Molecular Hydrogen Emission Line (MHEL) regions recently discovered towards the same outflow sources (Davis et al. 2001 - Paper I). The [FeII] and $\mathrm{H}_{2}$ lines both trace emission from the base of a large-scale collimated outflow, although they clearly trace different flow components. We find that the [FeII] is associated with higher-velocity gas than the $\mathrm{H}_{2}$, and that the [FeII] emission peaks further away from the embedded source in each system. This is probably because the $[\mathrm{FeII}]$ is more closely associated with $\mathrm{HH}$-type shocks in the inner, on-axis jet regions, while the $\mathrm{H}_{2}$ may be excited along the boundary between the jet and the near-stationary, dense ambient medium that envelopes the protostar. Indeed, there is spatial and kinematic evidence that [FeII] and the more typically-used optical emission lines, the red [SII] doublet, do trace almost the same shock-excited regions in $\mathrm{HH}$ jets and FEL regions alike.
\end{abstract}

Key words. ISM: jets and outflows - stars: pre-main-sequence - ISM: Herbig-Haro objects

\section{Introduction}

Comparitively little is known about Herbig-Haro (HH) jets and molecular outflows close to their protostellar driving sources. Optical forbidden-line emission from the base of $\mathrm{HH}$ jets from classical T Tauri stars (CTTS) has been studied with highresolution imaging (HST and ground-based $\mathrm{AO}$ ) and spectroscopic techniques (e.g. Ray et al. 1996; Bacciotti et al. 2000; Dougados et al. 2000; Hirth et al. 1994, 1997; Takami et al. 2001; Woitas et al. 2002), yet only recently have observations yielded information on the same regions in molecular outflows from more deeply embedded protostars (Davis et al. 2001 hereafter referred to as Paper I; Davis et al. 2002). The recent discovery of "Molecular Hydrogen Emission Line (MHEL)" regions in Class I young stellar objects (YSOs) is of considerable interest, since the survival of $\mathrm{H}_{2}$ in the inner jet regions constrains models of $\mathrm{HH}$ jet acceleration, collimation and evolution within a few hundred AU of these very young outflow sources.

The $\mathrm{H}_{2}$ echelle observations presented in Paper I reveal complex, low and high-velocity molecular line emission almost coincident with each Class I source. The characteristics of these MHEL regions are much like those of the "Forbidden Emission Line (FEL)" regions observed in CTTSs (e.g.

Send offprint requests to: C. J. Davis, e-mail: c.davis@jach.hawaii.edu
Hirth et al. 1997), even though the optical lines and near-IR $\mathrm{H}_{2}$ lines trace very different excitation conditions. In both MHELs and FELs: $i$ ) multiple (low and high) velocity components are observed; ii) the emission regions are generally offset from the source, along the blue-shifted flow axis, by a few tens to a few hundred AU; and iii) the higher-velocity gas is always slightly further offset than the slower gas. Indeed, even though molecular hydrogen is dissociated under extreme excitation conditions, radial $\mathrm{H}_{2}$ velocities approaching $50-150 \mathrm{~km} \mathrm{~s}^{-1}$ are observed in some MHEL regions.

To further investigate the relationship between MHEL and FEL regions, we now present spectroscopic observations made in [FeII] at $1.644 \mu \mathrm{m}$. The same Class I sources observed in $\mathrm{H}_{2}$ at $2.122 \mu \mathrm{m}$ (apart from GGD 27), as well as three CTTSs (Class II sources) known to possess optical FEL regions and $\mathrm{HH}$ jets, have been observed in [FeII] emission. [FeII] is a useful tracer of intermediate/high-excitation shocks in HH objects and molecular outflows. Much like the optical forbidden lines from, e.g. [OI], [SII], [NII], etc., the near-IR [FeII] lines can be used to complement $\mathrm{H}_{2}$ observations (e.g. Reipurth et al. 2000). In outflows the shock-excited $\mathrm{H}_{2}$ emission derives from dense $\left(10^{4} \mathrm{~cm}^{-3}\right)$, warm $(\sim 2000 \mathrm{~K})$ molecular gas, while the metastable transitions of species like [FeII] dominate the cooling at similar densities though higher temperatures, of the order of $10^{4} \mathrm{~K}$ (Hollenbach \& McKee 1989). Hamann et al. (1994) have used H-band [FeII] lines to probe FEL regions in 
Table 1. Observing log: the top seven targets are Class I YSOs; the bottom three are T Tauri stars.

\begin{tabular}{lcccc}
\hline \hline Source & $\begin{array}{c}\text { RA } \\
(2000.0)\end{array}$ & $\begin{array}{c}\text { Dec } \\
(2000.0)\end{array}$ & $\begin{array}{c}{ }^{1} \text { Slit } \\
\text { PA }\end{array}$ & $\begin{array}{c}{ }^{2} \text { Exp. } \\
\text { Time } \\
(\mathrm{min})\end{array}$ \\
\hline SVS 13 & 032903.7 & +311602 & $123^{\circ}$ & 40 \\
B5-IRS1 & 034741.6 & +325146 & $73^{\circ}$ & 33 \\
IRAS 04239+2436 & 042656.4 & +244336 & ${ }^{3} 59^{\circ}$ & 40 \\
L1551-IRS5 & 043133.6 & +180811 & $66^{\circ}$ & 33 \\
HH 34-IRS & 053529.8 & -062658 & $167^{\circ}$ & 33 \\
HH 72-IRS & 072008.4 & -240223 & $90^{\circ}$ & 40 \\
HH 379-IRS & 214508.2 & +473307 & $82^{\circ}$ & 40 \\
& & & & \\
DG Tau & 042704.7 & +260617 & $46^{\circ}$ & 40 \\
RW Aur & 050749.6 & +302405 & $125^{\circ}$ & 33 \\
AS 353A & 192031.0 & +110155 & $106^{\circ}$ & 40 \\
\hline
\end{tabular}

1 Slit position angle (east of north).

2 Total on-source exposure time.

3 A PA of $45^{\circ}$ was used in Paper I for our $\mathrm{H}_{2}$ observations of IRAS $04239+2436$. This new angle was measured from the HST images of Reipurth et al. (2000).

a handful of T Tauri stars. The same [FeII] lines should therefore be a good tracer of the FEL regions associated with Class I YSOs, where optical observations will be hampered by extinction.

\section{Observations}

Echelle spectra were obtained at the U.K. Infrared Telescope (UKIRT) on 1-3 November 2001 UT using the facility $1-5 \mu \mathrm{m}$ spectrometer CGS 4 (Mountain et al. 1990). Observations in the $a^{4} \mathrm{D}_{7 / 2}-a^{4} \mathrm{~F}_{9 / 2}$ transition of [FeII] at $\lambda_{\mathrm{vac}}=1.643998 \mu \mathrm{m}$ (Johansson 1978) were acquired towards seven Class I sources and three Class II (T Tauri) stars (listed in Table 1). CGS 4 is equipped with a $256 \times 256$ pixel InSb array; the pixel scale at $1.64 \mu \mathrm{m}$ measures $0 .{ }^{\prime} 41 \times 00^{\prime} 88\left(0 .{ }^{\prime} 41\right.$ in the dispersion direction). A 2-pixel-wide slit was used, resulting in a velocity scale of $\sim 8.0 \mathrm{~km} \mathrm{~s}^{-1}$ per pixel. The instrumental profile in the dispersion direction, measured from Gaussian fits to arc lines, was $19.0( \pm 1.0) \mathrm{km} \mathrm{s}^{-1}$. For each target the spectrometer slit was orientated along the outflow or $\mathrm{HH}$ jet axis, as defined by published, large-scale images of each system (the same slit position angles as in Paper I were used, except for IRAS 04239+2436 where a revised angle was employed based on newly published data).

A sequence, comprising one sky followed by three object exposures was repeated a number of times for each source to build up signal-to-noise, the sky position being typically $30^{\prime \prime}-60^{\prime \prime}$ away from the source (in a direction orthogonal to the flow axis). Each spectral image was bias subtracted and flatfielded. Sky-subtracted object frames were then co-added into reduced "groups" (one group frame per target). To wavelengthcalibrate these group images, argon and krypton arc lamp spectra were observed just prior to observing each source (and each standard star). The combined lamp spectral images yielded six lines, spread across the dispersion axis, that could be used for accurate wavelength calibration. The argon and krypton lines at $\lambda_{\mathrm{vac}}=1.644107 \mu \mathrm{m}$ and $1.647035 \mu \mathrm{m}$ were observed directly (in the 33rd order) while lines at $1.694521 \mu \mathrm{m}$ and $1.599386 \mu \mathrm{m}$ (argon) and $1.670137 \mu \mathrm{m}$ and $1.694043 \mu \mathrm{m}$ (krypton) were detected from adjacent (32nd or 34th) orders. The rest wavelength of [FeII] also lies close to the bright $\mathrm{OH}$ sky line, $\mathrm{OH}(5,3) \mathrm{R}_{1}(2)$ at $1.64421 \mu \mathrm{m}$ (Maihara et al. 1993); the separation between the [FeII] and $\mathrm{OH}$ lines is only $39 \mathrm{~km} \mathrm{~s}^{-1}$, or about 5 pixels. This sky-line did not subtract out perfectly in some cases, although because the line is narrow and extended along the full length of the slit (along whole columns), it was easily distinguished from the [FeII] emission associated with each outflow. In these cases, the line was fitted and removed from the spectral image. Before doing this, however, we used it to check the wavelength and subsequent velocity calibration (described below).

Routines available through Starlink were used to identify arc lines and wavelength-calibrate each spectral image, rowby-row. In this way we could also correct for curvature along the slit (spatial) axis in each image. The arc spectral images were first "self-calibrated" so that we could check for variations in the absolute wavelength calibration along the slit axis (along columns); these variations were found to be small, of the order of $5 \mathrm{~km} \mathrm{~s}^{-1}$ towards the edges of the array, and a factor of 2 better within the central $30 \%$ of the array where the [FeII] emission was observed. Instrument flexure over the duration of the observations could, however, introduce additional uncertainties in the absolute velocity calibration, i.e. by shifting the individual frames with respect to the wavelength reference used to calibrate the reduced group spectral image. By comparing the positions of sky lines in a number of raw frames we found that this effect was also small; indeed, the narrowness of the [FeII] lines and/or residual sky lines in the combined group data for each source (as compared to the instrumental profile width, which is measured from just one frame) confirms this finding. We therefore conclude that the overall velocity calibration is accurate to better than $6 \mathrm{~km} \mathrm{~s}^{-1}$, while perceived velocity shifts between adjacent spectra observed along the same slit will be more accurate, to within $2-3 \mathrm{~km} \mathrm{~s}^{-1}$.

Finally, bright G- or K-type giant or main-sequence stars were observed with the same instrumental configuration prior to each outflow source. Narrow telluric absorption features were evident at $1.6429 \mu \mathrm{m}$ and $1.6455 \mu \mathrm{m}$ in these data, lines which are displaced by $\sim 200 \mathrm{~km} \mathrm{~s}^{-1}$ and $\sim 220 \mathrm{~km} \mathrm{~s}^{-1}$ from the rest wavelength of the target [FeII] line. Some of the standard star spectra also possessed photospheric absorption features (from permitted FeI or other species) at $1.6427 \mu \mathrm{m}, 1.6437 \mu \mathrm{m}$, $1.6444 \mu \mathrm{m}$ and $1.6454 \mu \mathrm{m}$. These absorption lines were narrow $\left(\leq 25 \mathrm{~km} \mathrm{~s}^{-1}\right)$, so we were able to "patch" across them by interpolating between the continuum on either side of the line, before the standard star spectra were used to flux calibrate the extracted 1D source spectra.

\section{Results}

Our goal was to use [FeII] to trace FEL regions in the Class I YSOs where MHELs had already been observed. We therefore 
observed each target from Paper I (except GGD 27) using the same slit position angle (PA) and slit width, so that we could directly compare the $\mathrm{H}_{2}$ and [FeII] characteristics. In [FeII] we also observed three T Tauri stars. One of these, AS 353A, was observed in $\mathrm{H}_{2}$ in Paper I: the other two CTTSs have known FEL regions from published optical observations. With these CTTS data we can compare the optical and near-IR characteristics of the FELs, as traced in [SII] and [FeII] respectively. Note that shock models and observations of $\mathrm{HH}$ objects do indicate that $[\mathrm{FeII}]$ and $[\mathrm{SII}]$ trace similar post-shock regions (Hollenbach \& McKee 1989; Hamann et al. 1994), even though the [FeII] $1.64 \mu \mathrm{m}$ line has a higher critical density for collisional excitation than the $[\mathrm{SII}] \lambda 6716 / \lambda 6731$ lines $\left(3 \times 10^{4} \mathrm{~cm}^{-3}\right.$ as compared to $2 \times 10^{3} \mathrm{~cm}^{-3}$ for [SII]). The electron temperature in an [SII]-bright region is expected to be $\geq 13000 \mathrm{~K}$, while the $\mathrm{Fe}^{+}$ionisation fraction is predicted to peak at temperatures of approximately $14000 \mathrm{~K}$ (Hamann 1994); these temperature limits are not strongly dependent on the density (they were calculated for $n_{\mathrm{H}}=10^{5} \mathrm{~cm}^{-3}$ ).

[FeII] position-velocity $(P-V)$ diagrams for the seven Class I YSOs are shown in Fig. 1; the data obtained for the $\mathrm{T}$ Tauri stars are presented in Fig. 2. Where the FEL emission is obscured by strong continuum emission, we also show continuum-subtracted $P-V$ diagrams, in Fig. 3. In these plots the continuum emission on either side of the line emission has been fitted, row by row, with a 3 rd order polynomial. The fits are then subtracted to leave only the line emission associated with each target.

From the $P-V$ diagrams we also measure the relative positions of the FEL components with respect to the source continuum position. This is done by fitting Gaussians to profiles taken perpendicular to the dispersion direction. For each column in a given $P-V$ diagram (i.e. at each velocity) a measurement of the position of the line emission or the continuum emission is made (line emission positions are obtained from the continuum-subtracted $P-V$ diagrams). These are then plotted against velocity in Fig. 4. Because our spatial resolution along the slit is relatively poor with respect to the seeing, for error bars on the line emission positions (the boxes in Fig. 4) we use the Full Width Half Maximum ( $F W H M$ ) of the Gaussian fits divided by $\sqrt{(} N-1)$, where $N$ is the number of points across the profile $\left(=2 \times F W H M / 0.88^{\prime \prime}\right)$. The same technique was used in Paper I, where offsets of the MHEL components were presented (although note that the seeing was much better during the $\mathrm{H}_{2}$ observations). The position of the source continuum is known much more accurately and is indicated in each plot by a polynomial fit to the source continuum positions. The scatter in these continuum points about the fit is an indication of the accuracy with which the continuum position is known; this scatter is of course directly related to the strength of the continuum emission.

Spectra, representing in all cases the sum of 3 rows (equivalent to an on-source area of $\sim 0.8^{\prime \prime} \times 2.7^{\prime \prime}$ ) are shown inset in Figs. 1 and 2 for select positions in each region. We only present spectra for regions where complex or interesting line profiles were observed.

Finally, a comparison between these new [FeII] data and the $\mathrm{H}_{2}$ observations discussed in Paper $\mathrm{I}$ is made in Sect. 7 and Figs. 6 and 7. First, however, we describe the [FeII] results pertaining to each source separately.

\section{4. [Fell] spectroscopy of Class I sources}

\subsection{SVS $13(H H 7-11)$}

The HH7-11 outflow in NGC $1333(d \sim 220 \mathrm{pc})$ has been observed extensively at near-IR wavelengths (e.g. Garden et al. 1990; Stapelfeldt et al. 1991; Carr 1993; Gredel 1996; Chrysostomou et al. 2000; Khanzadyan et al. 2002). The HH objects themselves reside along the edges of a southeastern, blue-shifted cavity that is bounded by a fast-moving molecular shell (Bachiller \& Cernicharo 1990; Bachiller et al. 2000). The probable source of the flow, SVS 13, is known to have undergone an outburst in recent years (Eislöffel et al. 1991) and, from Paper I, is clearly associated with complex $\mathrm{H}_{2}$ line emission. Recently, SVS 13 was found to be a close-binary (separation $\sim 0.3^{\prime \prime}$ ), with an orbital period of $\sim 1700$ yrs (Anglada et al. 2000).

In Fig. 1a we show a $P-V$ plot of the region near the source. [FeII] line emission was not detected from the HH objects that were covered by the spectrograph slit (the slit extended $44^{\prime \prime}$ to the $\mathrm{SE}$, and $42^{\prime \prime}$ to the NW; it did not reach HH 7). However, [FeII] was detected towards SVS 13. An extracted spectrum is shown inset in Fig. 1a. The profile is double peaked and blue-shifted with respect to the systemic LSR velocity of $+8 \mathrm{~km} \mathrm{~s}^{-1}$ (Bachiller \& Cernicharo 1990), which is marked with a dashed line in the $P-V$ diagram. The stronger of the two [FeII] components peaks at $V_{\mathrm{LSR}} \sim-133( \pm 10) \mathrm{km} \mathrm{s}^{-1}$; the weaker component peaks at somewhere in the range -20 to $-50 \mathrm{~km} \mathrm{~s}^{-1}$. Both components appear to be quite broad; the FWHM of the stronger, higher-velocity component (HVC) measures $73( \pm 10) \mathrm{km} \mathrm{s}^{-1}$; deeper spectra are needed to establish the width of the weaker, marginally-detected, low-velocity component (LVC), although a two-component Gaussian fit to our data suggests a similar FWHM.

In Fig. 3a we show a continuum-subtracted $P-V$ diagram which shows the FEL region more clearly. By employing the same spectro-astrometric technique used in Paper I (and described above) we measure the position of the brighter [FeII] HVC with respect to the source continuum position (Fig. 4). We find that the HVC is coincident with the source, to within a conservative measurement error of $\sim 0.5^{\prime \prime}$, or $\sim 110 \mathrm{AU}$. Likewise, the $\mathrm{H}_{2}$ emission towards SVS 13 was found to be offset by less than $\sim 0.4^{\prime \prime}$ from the source (see Paper I).

\subsection{B5-IRS $1(\mathrm{HH} 366)$}

The embedded YSO B5-IRS $1(d \sim 350$ pc) drives an extensive east-west molecular outflow (Yu et al. 1999) that excites two distant groups of $\mathrm{HH}$ objects, $\mathrm{HH} 366 \mathrm{E}$ and $366 \mathrm{~W}$. Much of the emission from HH 366 is situated well beyond the range of our spectrograph slit (Bally et al. 1996). There is, however, $\mathrm{H}_{2}$ emission within $30^{\prime \prime}$ of the source in both lobes (Yu et al. 1999). The optical knot HH366E5 in the eastern, blue-shifted flow lobe lies along our spectrograph slit. Although high velocity 

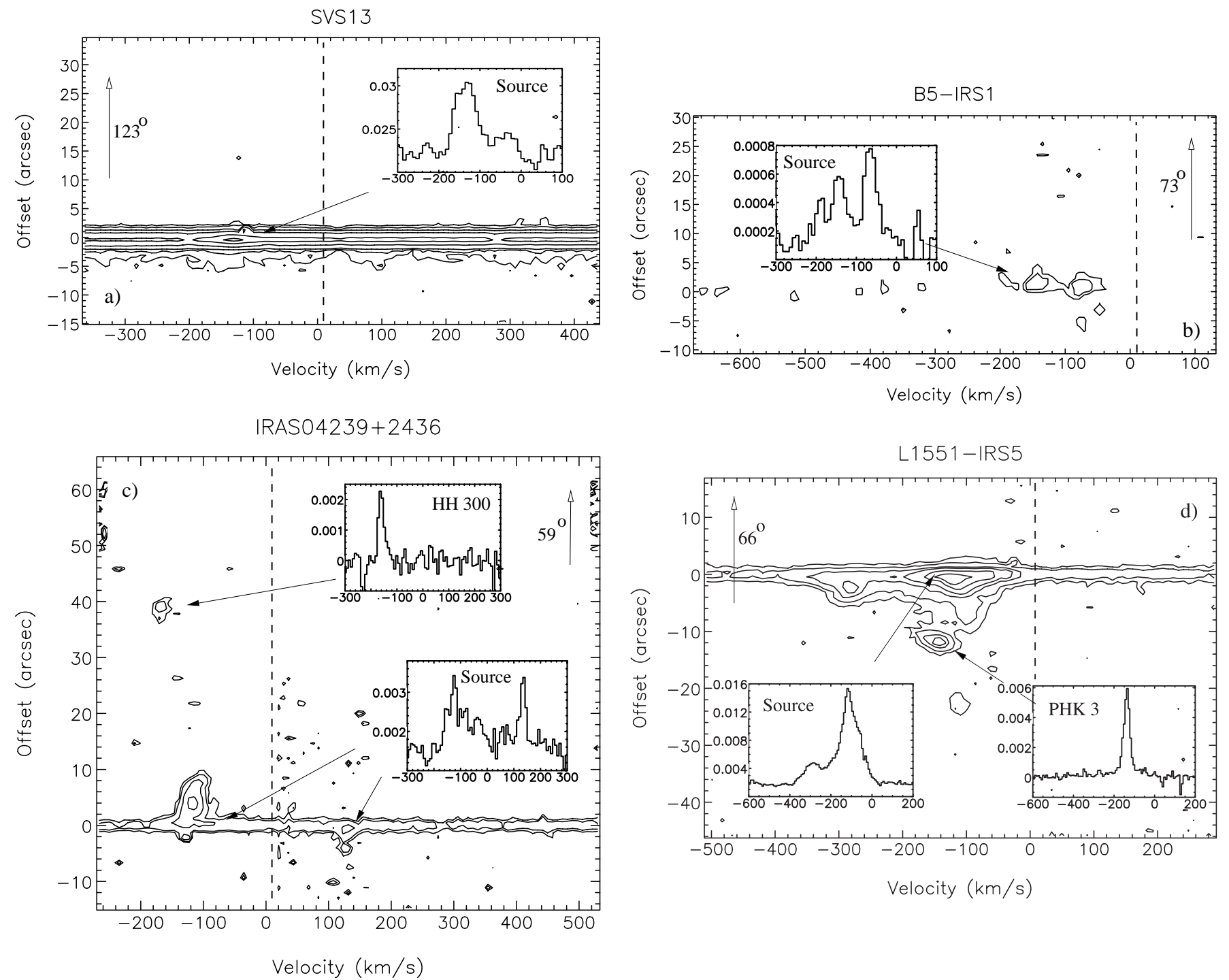

Fig. 1. [FeII] $1.644 \mu \mathrm{m} \mathrm{PV}$ plots for the Class I YSOs. $Y$-axis offsets (centred on the outflow source position) are in arcseconds along the slit. Vertical dashed lines mark the systemic LSR velocity in each region. Arrows show the slit orientation on the sky. In SVS 13 the contours measure 5, 10, 20,50,100 and 150× the standard deviation to the mean background, $\sigma$; in B5-IRS1 the contours are 2 and $4 \sigma ;$ in IRAS $04239+2348$ they are 3, 5, 10 and $20 \sigma$ and in L 1551-IRS5 they measure 5, 10, 20, 30, 50 and 75 $\sigma$. Extracted spectra (the sum of three rows in all cases) are superimposed; the axes on all spectra are in units of $\mathrm{km} \mathrm{s}^{-1}$ and Jy. Spectra are offset (from each source) by 0" in SVS 13, 0" in B5-IRS1, 0" and $38.7^{\prime \prime}$ in IRAS 04239+2348, and $0^{\prime \prime}$ and $-11.4^{\prime \prime}$ in L 1551-IRS5.

$\mathrm{H}_{2}$ emission was detected from this object (Paper I), we did not detect it in [FeII].

$\mathrm{H}_{2}$ and [FeII] emission are detected from the source position, though in both cases the emission is very weak (see Sect. 7 for a comparison). Overall, the [FeII] emission appears to be blue-shifted (with respect to the systemic velocity of $\sim 10 \mathrm{~km} \mathrm{~s}^{-1}$; Yu et al. 1999) to LSR velocities over a broad range from $\sim-50 \mathrm{~km} \mathrm{~s}^{-1}$ to $\sim-200 \mathrm{~km} \mathrm{~s}^{-1}$.

\subsection{IRAS $04239+2436(H H 300)$}

The low-luminosity Class I YSO IRAS 04239+2436 in Taurus $(d \sim 140 \mathrm{pc})$ drives a highly-collimated, knotty, $\sim 10^{\prime \prime}$-long [FeII] jet that has recently been observed in detail with NICMOS on HST (Reipurth et al. 2000). However, in these imaging data, within $1^{\prime \prime}$ of the source the [FeII] jet is obscured by the continuum emission from the star. The jet also probably drives a group of extensive optical HH bow shocks (HH 300 A-C; Reipurth et al. 1997) found about $30^{\prime}$ to the southwest, as well as a more compact conical HH feature, HH 300 D, about $40^{\prime \prime}$ to the northeast of the source.

The source itself, which is a binary (separation $\sim 0.3^{\prime \prime}$; Reipurth et al. 2000), is notable for having a rich near-IR spectrum (Green \& Lada 1996). We detect both $\mathrm{H}_{2}$ (Paper I) and $[\mathrm{FeII}]$ emission from the source continuum position (see Sect. 7). In the extracted [FeII] spectrum in Fig. 1c emission from both lobes of the flow is detected. The two jet lobes must therefore be traced to within an arcsecond of the star. Moreover, extinction does little to impede our detection of the jet and counterjet in [FeII] near the source. This suggests that the flow axis must be orientated close to the plane of the sky, and that the 

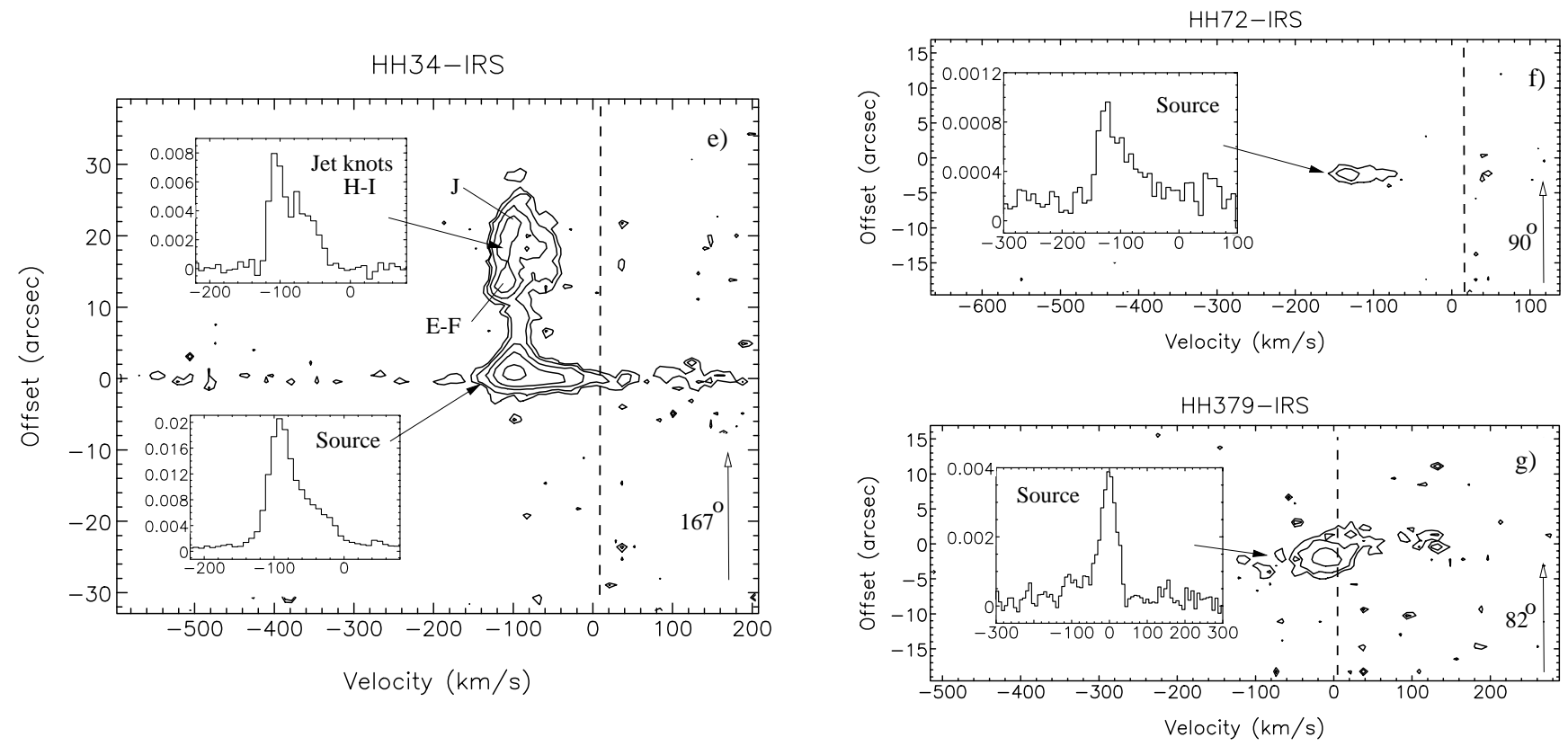

Fig. 1. Continued. In HH 34-IRS and HH 72-IRS the contours measure 3, 5, 10, 20 and 50× the standard deviation to the mean background, $\sigma$, while in HH 379-IRS they measure 5, 10 and 20 $\sigma$. Spectra are offset by $0^{\prime \prime}$ and 16.7" in HH 34-IRS, 0" in HH 72-IRS, and 0" in HH 379-IRS.

accreting, circumstellar disk must have a thickness that is less than our 3-pixel-wide extracted region $\left(\sim 2.7^{\prime \prime}\right.$, or $\left.<380 \mathrm{AU}\right)$.

The blue- and red-shifted [FeII] line-emission components in Fig. 1c are shifted to about the same radial absolute velocity with respect to the systemic rest velocity $\left(\sim 8 \mathrm{~km} \mathrm{~s}^{-1}\right)$; the peaks occur at LSR velocities of $-125( \pm 5) \mathrm{km} \mathrm{s}^{-1}$ and $+133( \pm 5) \mathrm{km} \mathrm{s}^{-1}$ respectively. The blue peak is also probably associated with a weaker, though much broader velocity component that extends from $V_{\mathrm{LSR}} \sim 0 \mathrm{~km} \mathrm{~s}^{-1}$ to $\sim-200( \pm 10) \mathrm{km} \mathrm{s}^{-1}$. The red counterjet peak is, on the other hand, extremely narrow (we measure a $F W H M$ of $\left.24( \pm 3) \mathrm{km} \mathrm{s}^{-1}\right)$; this line is only marginally broader than the instrumental profile.

For the $[\mathrm{FeII}]$ observations we used a revised slit PA of $59^{\circ}$ (an angle of $45^{\circ}$ was used for the $\mathrm{H}_{2}$ spectroscopy in Paper I), which is better aligned with the jet axis. In [FeII] we trace the entire $\sim 10^{\prime \prime}$-long blue-shifted jet lobe, as well as spatiallyextended red-shifted emission from the counter-jet. These jet components are most clearly seen in the continuum-subtracted $P-V$ plot in Fig. 3b. The [FeII] line emission is strongest at an offset of $4^{\prime \prime}\left( \pm 0.5^{\prime \prime}\right)$ along the northeastern, blue-shifted jet lobe. This emission peak probably corresponds to the bright [FeII] knots J3-J5 in the HST images of Reipurth et al. (2000). In Fig. 4 we again plot the positions of these emission peaks with respect to the source continuum position. The $2^{\prime \prime}-5^{\prime \prime}$ offsets in the blue lobe reflect the fact that the apparent [FeII] intensity increases with distance from the source. Extinction near the source is probably not the cause of this increase, since the red-shifted counterjet emission peaks closer to the source in Fig. $4 \mathrm{~b}$, at offsets of only $\sim 1^{\prime \prime}$. Instead, the [FeII] emission must be enhanced along the jet axis, probably in discrete HH-type shocks. We also note in Fig. 4b that the higher-velocity blueshifted $[\mathrm{FeII}]$ emission appears closer to the source. In other words, we see an apparent decrease in offset with velocity.
Finally, in addition to [FeII] emission from the source and inner jet (FEL) region, we also detect [FeII] at a distance of about $38^{\prime \prime}$ to the northeast of the source. This emission is presumably associated with HH 300D (Reipurth et al. 1997). The [FeII] peak shown in Fig. 1c is blueshifted to an LSR velocity of $-167( \pm 5) \mathrm{km} \mathrm{s}^{-1}$, though the emission line is also extremely narrow $\left(F W H M=25( \pm 3) \mathrm{km} \mathrm{s}^{-1}\right)$; this suggests that the [FeII] is associated with high-velocity jet material, though with a relatively low-velocity shock within the flow.

\subsection{1551-IRS5}

L 1551-IRS5 is an archetypal bipolar molecular outflow that can be observed at relatively high spatial resolution because of its close proximity to the earth $(d \sim 140 \mathrm{pc})$. The flow consists of a striking blue-shifted, wind-swept cavity that extends over $10^{\prime}(0.5 \mathrm{pc})$ to the southwest of IRS 5 (Stocke et al. 1988; Moriarty-Schieven \& Snell 1988). The cavity is associated with an array of optical and near-IR shock features (e.g. Mundt \& Fried 1983; Davis et al. 1995). The source itself (which is a binary system) appears to drive two small-scale jets (Fridlund \& Liseau 1998; Hartigan et al. 2000; Itoh et al. 2000; Davis et al. 2002). However, these jet-like features may instead represent the edges of a single collimated flow (Mundt et al. 1991; Pyo et al. 2002). Indeed, the new images of Pyo et al. represent perhaps the strongest evidence yet that the two "jets" are simply the limb-brightened edges of a small, ovoidal cavity, that is bounded at its leading edge by a bright bow, which they label PHK 3.

In $\mathrm{H}_{2}$ we detected complex velocity structure along the L 1551-IRS5 jet (Paper I). Likewise, in [FeII], multicomponent profiles are again observed, though in detail the $\mathrm{H}_{2}$ and $[\mathrm{FeII}]$ emissions are very different. As we shall discuss further in Sect. 7, the LVC observed in $\mathrm{H}_{2}$ is not detected in 
[FeII]; in the latter, only an HVC and what we shall refer to as an "extremely-high-velocity component" (EHVC) is observed. The HVC and EHVC in Fig. 1d peak at $-120( \pm 5) \mathrm{km} \mathrm{s}^{-1}$ and $-285( \pm 15) \mathrm{km} \mathrm{s}^{-1}$ respectively (both are strongly blueshifted with respect to the systemic velocity of $6 \mathrm{~km} \mathrm{~s}^{-1}$; MoriartySchieven \& Snell 1988). Both components are also quite broad; a two-component Gaussian fit yields FWHM widths of $113( \pm 5) \mathrm{km} \mathrm{s}^{-1}$ and $125( \pm 15) \mathrm{km} \mathrm{s}^{-1}$ for the HVC and EHVC. The overall $F W Z I$ range in radial velocities observed in [FeII] is also roughly equal to those seen in [SII] (Hartigan et al. 2000).

In Figs. 3 and 4 we again show a continuum-subtracted $P-V$ diagram and a plot of the positions of the line-emisson peaks with respect to the source continuum. Spatially, the HVC peak is closer to the source continuum position than the EHVC; the EHVC is offset by about $2^{\prime \prime}-3^{\prime \prime}$ to the southwest, about twice as far as the HVC along the blue-shifted flow axis. However, although the EHVC peak is offset further downwind, overall the HVC emission extends almost twice as far along the flow. We also note once again an apparent decrease in offset with velocity in Fig. 4c, when considering the EHVC separately. Very similar results were recently reported by Pyo et al. (2002).

Further downwind, we detect compact [FeII] peaks at offsets of $\sim 12^{\prime \prime}$ and $\sim 23^{\prime \prime}$. In velocity space both peaks are narrow; for the brighter feature we measure a FWHM of $40( \pm 3) \mathrm{km} \mathrm{s}^{-1}$. This bright peak is blue-shifted to an intermediate velocity of $-140( \pm 10) \mathrm{km} \mathrm{s}^{-1}$. Both features correspond to discrete knots along the jet axis. The feature at $12^{\prime \prime}$ coincides with the optical/[FeII] bow shock PHK 3 (labeled knot D by Fridlund \& Liseau 1998).

\subsection{HH34-IRS}

The HH 34 outflow in L $1641(d \sim 450 \mathrm{pc})$ is part of a parsecscale "superjet" that includes HH 33, 40 and 85 to the north, and HH 86-88 to the south (Devine et al. 1997a). The collimated, knotty, HH 34 jet itself has been the subject of intense scrutiny at optical and near-IR wavelengths. Within $22^{\prime \prime}$ of the IRS source, the jet remains highly collimated, the optical emission being confined to within a width of less than $1^{\prime \prime}$ (Ray et al. 1996; Reipurth et al. 2000). The flow is orientated at an angle of $\sim 23^{\circ}$ to the plane of the sky, and in optical emission lines radial and tangential jet-knot velocities of $\sim-100 \mathrm{~km} \mathrm{~s}^{-1}$ and $150-300 \mathrm{~km} \mathrm{~s}^{-1}$ have been recorded (Bührke et al. 1988; Heathcote \& Reipurth 1992; Eislöffel \& Mundt 1992).

Like L 1551-IRS5, the HH34 jet near the source is observed in both $\mathrm{H}_{2}$ and [FeII] emission (the latter was first detected in the images of Stapelfeldt et al. 1991), though there are again differences in the details (discussed in Sect. 7). The [FeII] flux distribution closely follows that seen in [SII] (Ray et al. 1996), being much weaker within the first $10^{\prime \prime}$ of the source. The [FeII] profiles along the jet, at offsets between $10^{\prime \prime}$ and $20^{\prime \prime}$, comprise a blue-shifted peak at $V_{\mathrm{LSR}} \sim-120 \mathrm{~km} \mathrm{~s}^{-1}$ plus an extended red-wing. These broad lines appear almost double-peaked between knots E and I (Fig. 1e), although the [FeII] profiles furthest from the source (towards knot J) converge to a single, slightly less blue-shifted radial velocity of about $-100 \mathrm{~km} \mathrm{~s}^{-1}$. The [FeII] profiles are very similar to those seen in [SII], in terms of the velocity at the emission peak, the overall range of radial velocities observed, and the general "inverted-V" shape of the emission profile in the $P-V$ plot between knots E and J (Bührke et al. 1988; Heathcote \& Reipurth 1992).

Towards the continuum source position the [FeII] profile comprises a sharp peak at $-95( \pm 10) \mathrm{km} \mathrm{s}^{-1}$ and a broad red wing that extends back almost to the systemic rest velocity of $\sim 8 \mathrm{~km} \mathrm{~s}^{-1}$ (Chernin \& Masson 1995). The bulk of the [FeII] emission towards HH34-IRS is therefore blue-shifted to velocities that are almost as high as those seen along the jet.

In Paper I we found that the $\mathrm{H}_{2}$ emission towards HH34IRS is coincident with the source continuum position (unlike the other YSOs observed, where the $\mathrm{H}_{2}$ towards the source is usually offset along the blue-shifted flow lobe by at least a few tenths on an arcsecond). Unfortunately, we detected only very weak continuum emission from $\mathrm{HH} 34$-IRS in our $H$-band observations, so we cannot measure (or set accurate upper limits on) the offset between the [FeII] line emission and the continuum position.

\section{6. $H H 72-I R S$}

The distant, intermediate-mass YSO HH 72-IRS $(d \sim 1500 \mathrm{pc})$ in L 1660 drives an east-west bipolar molecular outflow (Schwartz et al. 1988; Reipurth \& Graham 1988). No collimated $\mathrm{HH}$ jet is observed from this YSO; optical HH emission knots are only observed near the eastern end of the flow, where it exits the dense core that harbours the powering source. However, additional shock features are detected closer to the source in $\mathrm{H}_{2}$ emission (Davis et al. 1997, 2002). From the partial overlap between the CO flow lobes, this outflow is probably orientated at $30^{\circ}-60^{\circ}$ to the plane of the sky.

Unpublished [FeII] images obtained by one of us (CJD) reveal emission only from the optical HH 72 bow shock (the knots labelled A, B and C by Reipurth \& Graham 1988). Our spectrograph slit, which was aligned along the small-scale $\mathrm{H}_{2}$ jet axis that extends a few arcseconds to the east of the IRS source (Davis et al. 2002), passed just to the north of these [FeII] features, so they do not appear in the $P-V$ plot in Fig. 1f. However, [FeII] emission was detected towards the source continuum position. The [FeII] profile in Fig. 1f is very similar to the profile seen towards HH34-IRS, even though it is much weaker and is associated with a more distant and more massive YSO. The [FeII] emission from HH 72-IRS peaks at $V_{\mathrm{LSR}} \sim-130( \pm 15) \mathrm{km} \mathrm{s}^{-1}$, while a broad red wing extends to near-zero radial velocities (with respect to the systemic rest velocity of $+20 \mathrm{~km} \mathrm{~s}^{-1}$; Schwartz et al. 1988).

As with HH 34-IRS, we are not able to accurately measure the offset between the line emission and the source continuum because the latter is too weak in the $H$-band.

\subsection{HH379-IRS}

The HH 379 outflow is situated near the molecular cloud 093.5-04.3 in Cygnus (Dobashi et al. 1994) at a distance of 

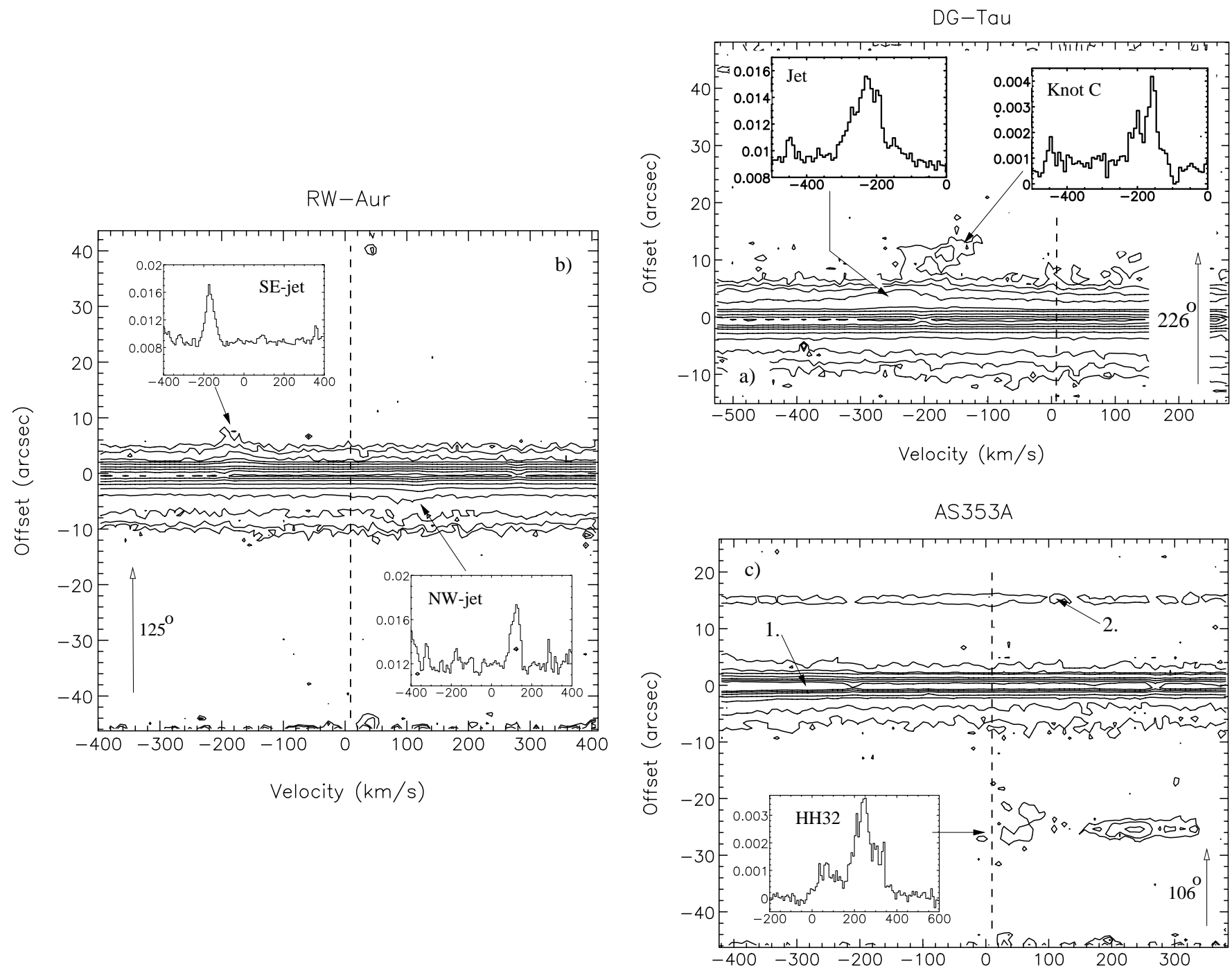

Fig. 2. Same as Fig. 1, except that in AS 353A the contours measure 5, 10, 20, 50, 100, 150, 200, 400, 800 $\sigma$, while in DG Tau and RW Aur the contours represent $3,5,10,20,50 \ldots$ (etc.) $\sigma$. Spectra are offset by $3.5^{\prime \prime}$ and $11.4^{\prime \prime}$ in DG Tau, 2.6" and $-2.6^{\prime \prime}$ in RW Aur, and $-24.6^{\prime \prime}$ in AS 353A.

$\sim 0.9$ kpc. HH379-IRS may be associated with a nearby compact optical nebula (Devine et al. 1997b). In Paper I we assumed that this conical nebula harbours the HH energy source and therefore positioned our spectrograph slit through the nebula and the $\mathrm{HH}$ object; we have done the same here with our [FeII] observations.

No continuum emission was detected from the outflow source in the [FeII] data. However, a distinct [FeII] lineemission peak is observed towards the nominal source position (Fig. 1g). The emission from HH379-IRS is morphologically and kinematically very similar in [FeII] and $\mathrm{H}_{2}$. Both $\mathrm{H}_{2}$ and [FeII] peaks in the $P-V$ diagrams appear to be elongated at a position angle that implies a blue-shifted flow towards the west of the source and a red-shifted flow to the east. However, note that HH 379-IRS is somewhat unique amongst the Class I YSOs observed here, since it is the only source with [FeII] that peaks near the systemic rest velocity (discussed further in Sect. 7).
Again, we do not measure the offset between the line emission and the IRS source position because of the lack of continuum emission at these shorter wavelengths.

\section{5. [Fell] spectroscopy of classical T Tauri stars}

DG Tau and RW Aur were not part of our original $\mathrm{H}_{2}$ survey (Paper I), although AS 353A was observed in the earlier study. We discuss these three sources separately below.

\subsection{DG Tau (HH 158)}

The classical T Tauri star DG Tau $(d \sim 140 \mathrm{pc})$ drives a wellcollimated HH jet (HH 158) that extends over about $10^{\prime \prime}$ to the southwest (Mundt et al. 1987; Lavalley et al. 1997; Eislöffel $\&$ Mundt 1997). The jet itself has recently been observed at high spatial resolution, with HST by Bacciotti et al. (2000, 2002) and with adaptive optics (AO) by Dougados et al. (2000). 

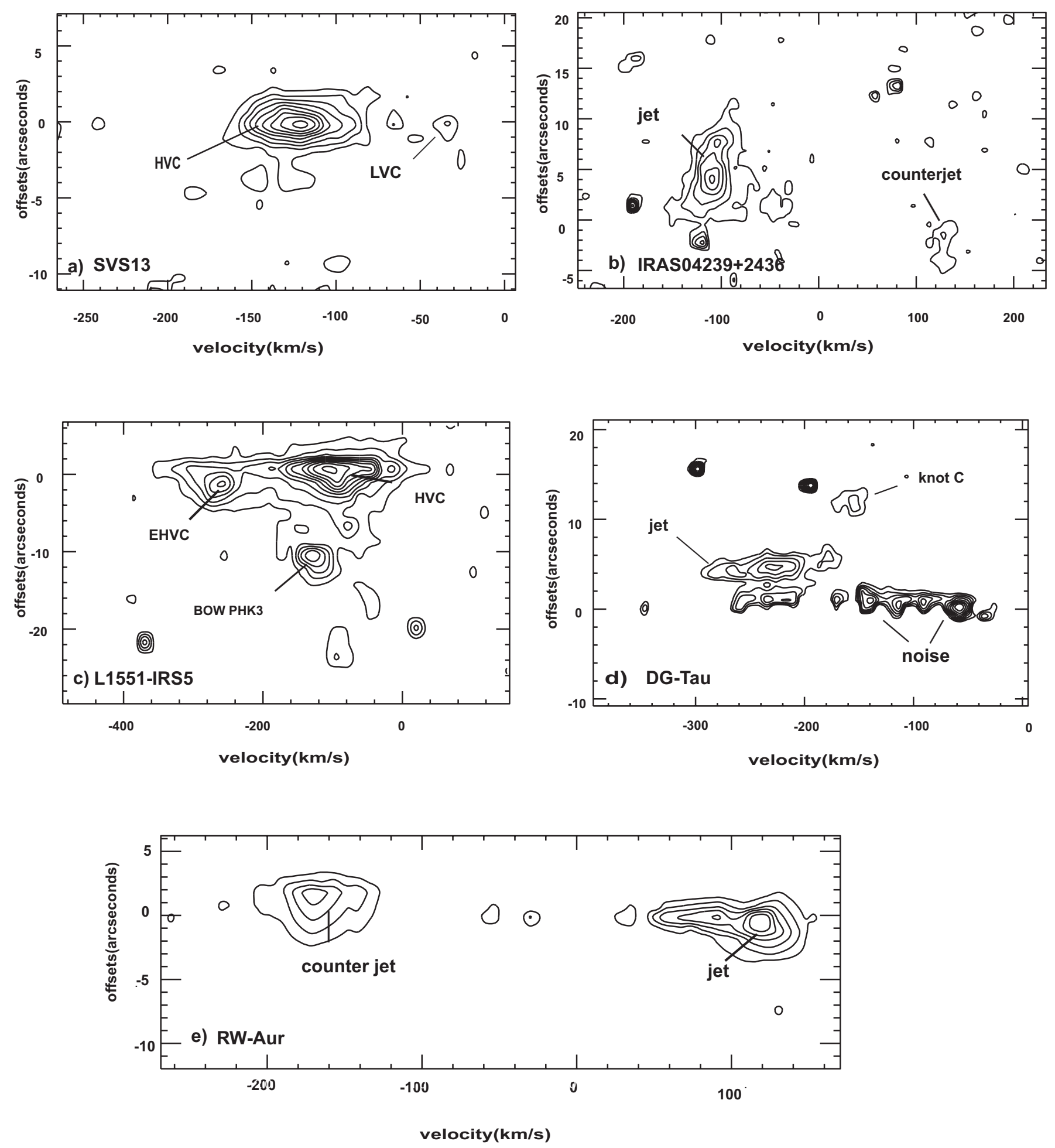

Fig. 3. [FeII] $P-V$ diagrams of five of the outflows. Only emission within approximately $15^{\prime \prime}$ of each YSO is plotted. The continuum emission from each source has been fitted and removed. Contours start at the $3 \sigma$ background (blank sky) noise level and increase by a factor of $\sqrt{2}$.

These data show the jet structure within a few arcseconds of the source, and even point to possible precession and/or jet-rotation on arcsecond scales. Here we adopt the same slit PA as was used by Bacciotti et al. (1998) in their HST STIS observations.

Hamann et al. (1994) report the detection of blue-shifted [FeII] $1.644 \mu \mathrm{m}$ emission from DG Tau, though they did not observe along the jet axis. Takami et al. (2002) show
[FeII] $1.257 \mu \mathrm{m}$ spectra which likewise exhibit high, blueshifted velocities, though again their spectrograph slit was not aligned with the jet axis. Here we detect [FeII] predominantly from the DG Tau jet (although we do not detect a counterjet). In these data, within $1^{\prime \prime}-2^{\prime \prime}$ of the source the [FeII] peaks at $V_{\mathrm{LSR}}=-235( \pm 10) \mathrm{km} \mathrm{s}^{-1}$ (this spectrum is labelled "jet" in Fig. 2a) while further downwind (offset $-11.4^{\prime \prime}$ ), towards 

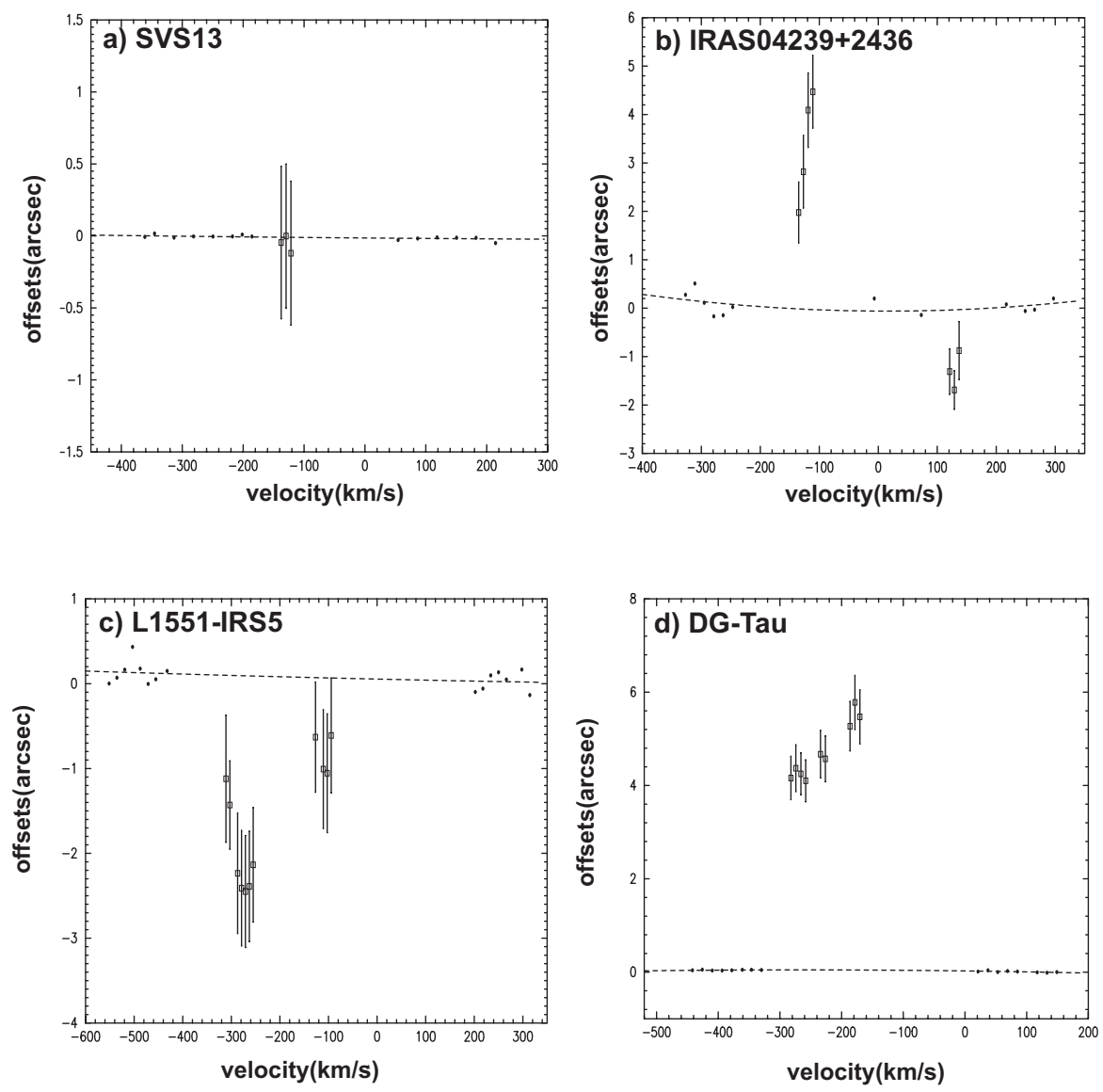

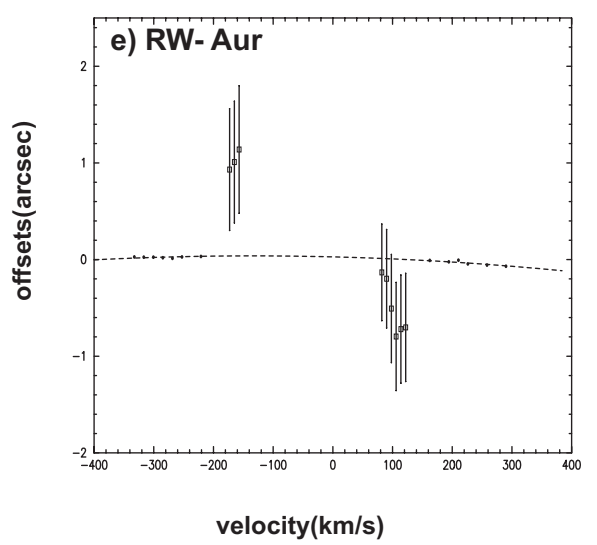

the bow-shock-like knot labelled knot $\mathrm{C}$ by Eislöffel \& Mundt (1997), the [FeII] peaks at $V_{\mathrm{LSR}}=-150( \pm 10) \mathrm{km} \mathrm{s}^{-1}$. Note that for DG Tau the systemic LSR velocity is $6.0 \mathrm{~km} \mathrm{~s}^{-1}$ (Kitamura et al. 1996) and that the weak features at $-460 \mathrm{~km} \mathrm{~s}^{-1}$ in the two spectra in Fig. 2a represent the same artefact, namely an imperfectly-subtracted sky line.

The decrease in velocity between the source and bow shock $\mathrm{C}$ in the DG Tau jet is continuous along the weaker [FeII] emission observed between these two features. The [FeII] is broad near the source $\left(F W H M \sim 100( \pm 5) \mathrm{km} \mathrm{s}^{-1}\right)$ though the profile narrows further downwind. The [FeII] radial velocities compare very closely with published [SII] observations (Mundt et al. 1987; Bacciotti et al. 2000).
Fig. 4. Offsets of the [FeII] emission peaks from the stellar continuum centroid. The boxes (with error bars) represent the [FeII] positions, measured in each column (at each velocity bin) from the $P-V$ diagrams. The points at high (blue- and red-shifted) velocities are measures of the continuum position. The dashed line in each plot is a polynomial fit to these continuum points; these lines show the source continuum position.
In Fig. 3d we show a continuum-subtracted $P-V$ diagram which more clearly shows the [FeII] emission near DG Tau. In Fig. 4d we plot the positions of these [FeII] emission peaks with respect to the source continuum. As with some of the Class I sources, we see that the offset of the FEL emission decreases with increasing blue-shifted velocity.

High-resolution optical observations of the DG Tau jet, in [SII] and [NII], reveal a compact knot at a projected distance of about $0.6^{\prime \prime}-0.8^{\prime \prime}$ from the source and a broader bow shock feature at a distance of $3^{\prime \prime}-4$ " (labelled A1 by Bacciotti et al. 2000). Based on the offsets recorded in Fig. 4d we associate the $[\mathrm{FeII}]$ emission with this bow shock. Excitation in a bow shock would certainly explain the broad emission-line profile 
shown in Fig. 2a. The FWZI of the profile points to a high shock velocity, of the order of $200 \mathrm{~km} \mathrm{~s}^{-1}$. If this bow shock is an "internal working surface", associated with faster jet material catching up with slower jet gas, then the velocity difference between these two outflow episodes must be very high.

Within 1" of DG Tau we do not detect any [FeII] emission. The knots in Fig. 3d (labelled "noise") are due to Shot noise associated with the bright source continuum emission; further along the slit, where the data are read-noise limited, noise levels are lower so faint [FeII] emission can be detected. Thus, any similarly weak [FeII] associated with the [SII] knot observed within $1^{\prime \prime}$ of the source (knot A2; Bacciotti et al. 2000) could easily be lost in this noise.

\subsection{RW Aur (HH229)}

RW Aur is a complex multiple star system located in TaurusAurigae $(d \sim 140 \mathrm{pc})$. The HH 229 jet is associated with the brightest stellar component "A", a classical T Tauri star which may itself be a spectroscopic binary (Gahm et al. 1999). The fainter components " $B$ " and " $C$ " form a close binary system (separation $0.12^{\prime \prime}$ ) that is situated about 1.5 " away. The lobes of the bipolar jet from RW Aur-A (hereafter referred to as simply RW Aur) are visible over a considerable distance; Mundt \& Eislöffel (1998) report a jet length of $\sim 145^{\prime \prime}$. In optical forbidden lines, radial velocities of $100-200 \mathrm{~km} \mathrm{~s}^{-1}$ have been observed along the central $20^{\prime \prime}$ of the jet (Hirth et al. 1994; Bacciotti et al. 1996). Recently, this region of the jet has been observed at high spatial resolution, using AO imaging (Dougados et al. 2000) and HST STIS spectroscopy (Woitas et al. 2002). Woitas et al. estimate an inclination angle for the jet of $<37^{\circ}$ to the plane of the sky.

In $[\mathrm{FeII}]$ we detect emission from both lobes of the bipolar jet (Figs. 2b and 3e). Again, the emission peaks are offset along the jet axis, by $0.5^{\prime \prime}-1.0^{\prime \prime}$ with respect to the source continuum centroid (Fig. 4e), with the red-shifted [FeII] emission peaking closest to the source. AO and HST STIS observations of RW Aur show that, in the optical, both lobes of the jet are knotty, though well collimated $\left(F W H M<0.6^{\prime \prime}\right.$; Dougados et al. 2000; Woitas et al. 2002). Woitas et al. find that the [SII] flux along the blue-shifted jet lobe is rather evenly distributed within $\sim 2^{\prime \prime}$ of RW Aur; in [FeII] the emission is also extended over this same region (Fig. 3e). In the red-shifted counterjet, however, the flow appears more knotty on subarcsecond scales in the [SII] data. We do not have the spatial resolution to resolve these knots in [FeII], though we do see emission along the length of this [SII] counterjet. We also note that, as in [SII], the red jet lobe is more extended at higher velocities (between $V_{\mathrm{LSR}} \sim 100 \mathrm{~km} \mathrm{~s}^{-1}$ and $160 \mathrm{~km} \mathrm{~s}^{-1}$ ) than it is at lower velocities $\left(V_{\mathrm{LSR}} \sim 50-100 \mathrm{~km} \mathrm{~s}^{-1}\right.$; see Fig. 3e). Moreover, in Fig. $4 \mathrm{e}$ there is some evidence that the [FeII] peak is slightly further offset at higher (red-shifted) velocities.

From the [FeII] profiles in Fig. 2b we measure peak radial LSR velocities of $-175( \pm 10) \mathrm{km} \mathrm{s}^{-1}$ and $+150( \pm 10) \mathrm{km} \mathrm{s}^{-1}$ for the jet and counterjet features (the systemic LSR velocity is $\sim 6 \mathrm{~km} \mathrm{~s}^{-1}$; Ungerechts \& Thaddeus 1987). On similar spatial scales, Hirth et al. (1994) measure [SII] radial velocities for the southeastern jet and northwestern counterjet of $-190 \mathrm{~km} \mathrm{~s}^{-1}$ and $+100 \mathrm{~km} \mathrm{~s}^{-1}$ respectively (note that we use the same slit PA). The [FeII] line profiles in Fig. 2b are narrow, however: Gaussian fits yield line widths at FWHM of $\sim 50 \mathrm{~km} \mathrm{~s}^{-1}$ for both lobes in RW Aur. We do not detect any [FeII] emission at low radial velocities, in either jet lobe (the [SII] emission is weaker, though still observed, at these low radial velocities). In other words, no LVC is detected in [FeII] in either lobe. This current lack of an LVC in FEL emission was also noted by Woitas et al. (2002) in their optical [SII] and [OI] observations. These authors have suggested that the LVC may well be variable on a timescale of a few years.

The highly blue- and red-shifted radial velocities evident in the [FeII] spectra indicate that the emission must be associated with fast-moving gas along the RW Aur flow axis. However, as with some of the other sources discussed above, the narrow line widths point to low shock velocities. Higher shock velocities are predicted for DG Tau (discussed above), where the $[\mathrm{FeII}]$ profiles are much wider (note that in HST images the bow shock feature A1 in DG Tau is laterally more extended than the knots in DG Tau; i.e. it has more extended wings).

\subsection{AS $353 A(H H 32)$}

The classical T Tauri star AS 353A $(d \sim 300 \mathrm{pc}$, Mundt et al. 1983; Eislöffel et al. 1990) drives an obliquely-viewed bipolar HH flow, known as HH 32 (Hartigan et al. 1986; Davis et al. 1996; Curiel et al. 1997). Optical and near-IR images and spectroscopy of the leading, redshifted HH 32 bow shock are convincingly modelled if the flow is inclined at an angle of $\sim 60^{\circ}$ to the plane of the sky (Solf et al. 1986; Hartigan et al. 1987; Davis et al. 1996).

As was the case in Paper I, two stars were detected along the single slit position observed in the AS 353A region, which we again label 1 and 2 in Fig. 2c (AS 353A itself, the apparent source of the bipolar HH 32 outflow, is referred to as star 1; note, however, that star 2 is not $\mathrm{AS} 353 \mathrm{~B}) . \mathrm{H}_{2}$ emission was detected from star 2, and not from star 1; we did not detect [FeII] from either.

We do detect spatially-compact [FeII] from the leading edge of the HH 32 bow shock. As expected, the [FeII] profile is red-shifted to very high radial velocities; the double-peaked profile in Fig. 2c comprises components at $+70( \pm 20) \mathrm{km} \mathrm{s}^{-1}$ and $+235( \pm 10) \mathrm{km} \mathrm{s}^{-1}$ while, overall, the emission extends over FWZI $\sim 400 \mathrm{~km} \mathrm{~s}^{-1}$ (the systemic LSR rest velocity is at $\sim 8 \mathrm{~km} \mathrm{~s}^{-1}$; Edwards \& Snell 1982). The [FeII] profile is again quite similar to its optical counterpart in [SII], where double-peaked lines with components at $\sim+60 \mathrm{~km} \mathrm{~s}^{-1}$ and $+270( \pm 10) \mathrm{km} \mathrm{s}^{-1}$ have been reported (Hartigan et al. 1987). However, in the [SII] spectra discussed by Hartigan et al. the low-velocity component is much stronger than the highvelocity peak; in our [FeII] data, we see the opposite behaviour. This is because the optical profile is summed across the whole HH 32 clumpy bow shock region, while our single slit passes through only the centre of the bow (it largely bypasses the limbbrightened bow shock wings, seen clearly and labelled knots B and $\mathrm{C}$ in high-resolution optical images; e.g. Curiel et al. 1997). 
Observing more of the emission from the bow wings would certainly explain the "enhanced" lower-velocity component in the [SII] profile. The double-peaked [FeII] profile in Fig. 2c, and the low-velocity $\mathrm{H}_{2}$ reported in Paper I, do therefore comply with previously published bow shock model fits to kinematic studies of HH 32 (e.g. Hartigan et al. 1987; Davis et al. 1996).

\section{Br12 observations}

The Br12 HI recombination line $\left(\lambda_{\mathrm{vac}}=1.641168 \mu \mathrm{m}\right)$ was included within the wavelength coverage of our echelle observations. The difference between the Br12 and [FeII] rest wavelengths is $-0.00283 \mu \mathrm{m}$, which is equivalent to a velocity shift of $516 \mathrm{~km} \mathrm{~s}^{-1}$. Br12 was detected in two Class I sources and in all three $\mathrm{T}$ Tauri stars. These data are presented in Fig. 5, with the rest wavelength of $\operatorname{Br} 12$ set to $V_{\mathrm{LSR}}=0 \mathrm{~km} \mathrm{~s}^{-1}$.

In SVS 13 the Br12 spectrum is broad and possibly slightly asymmetric (Fig. 5a). Gaussian fitting yields a $F W H M$ of $-181( \pm 10) \mathrm{km} \mathrm{s}^{-1}$ and a peak velocity of $-2( \pm 4) \mathrm{km} \mathrm{s}^{-1}$, although the actual intensity peak is shifted to about $-20 \mathrm{~km} \mathrm{~s}^{-1}$. A similarly broad, low-velocity line is observed towards the only other Class I YSO that we detected in Br12 emission, IRAS $04239+2436$ (Fig. 5b). Fits yield a $F W H M$ of $239( \pm 18) \mathrm{km} \mathrm{s}^{-1}$ and a peak velocity of $+3( \pm 9) \mathrm{km} \mathrm{s}^{-1}$ for this source.

Br12 emission was detected towards all three T Tauri stars (Figs. 5c-e), where the integrated line luminosities were at least $5 \times$ stronger than they were for the Class I YSOs. In DG Tau, the Br12 profile is blended with blue-shifted [FeII] emission, which in the rest frame of the HI line appears at high $\left(>250 \mathrm{~km} \mathrm{~s}^{-1}\right)$ radial velocities (indicated in Fig. $5 \mathrm{c}$ ). The Br12 is, nevertheless, again very broad and centred near the systemic rest velocity. The RW Aur Br12 profile in Fig. 5d is markedly asymmetric and strongly blue-shifted. The line consists of a central peak at $-95( \pm 5) \mathrm{km} \mathrm{s}^{-1}$ and broad blue and redshifted line wings. The profile extends over about $600 \mathrm{~km} \mathrm{~s}^{-1}$ FWZI. In AS 353A we observe very strong Br12 emission. The profile towards this source is notable for being extremely symmetric and Gaussian in shape, with no sign of enhanced linewing emission, nor blue or red-shifted absorption features. Overall, the width of the AS 353A profile (FWHM) measures $274( \pm 6) \mathrm{km} \mathrm{s}^{-1}$; the line also peaks at a very low radial velocity of $+16( \pm 2) \mathrm{km} \mathrm{s}^{-1}$.

HI observations of many of our targets were also presented in Paper I, where the $K$-band $\mathrm{Br} \gamma$ emission line was observed. The same slit positions and slit angles were used for the $\mathrm{Br} \gamma$ data as were used for $\mathrm{Br} 12 /[\mathrm{FeII}]$ and $\mathrm{H}_{2}$. $\mathrm{Br} \gamma$ was detected towards four Class I YSOs; SVS 13, IRAS 04239+2436, HH $34-$ IRS and GGD 27(1), as well as towards the only T Tauri star studied in Paper I, AS 353A. Here we detected Br12 in the same Class I sources, except for HH34-IRS and GGD27 (the latter was not observed at $1.64 \mu \mathrm{m}$ ). Our Br12 non-detection for $\mathrm{HH} 34-\mathrm{IRS}$ was not unexpected since the $\mathrm{Br} \gamma$ line was very weak in this source and, for the other Class I sources, the Br12 emission was $120-160 \times$ weaker than $\operatorname{Br} \gamma$. For AS 353A, the ratio is lower, $\operatorname{Br} \gamma / \operatorname{Br} 12 \sim 35$, because of the reduced extinction to this source. Indeed, extinction is probably the main cause of the differences in line ratios observed. For $T_{\mathrm{e}} \sim 10^{4} \mathrm{~K}$ and
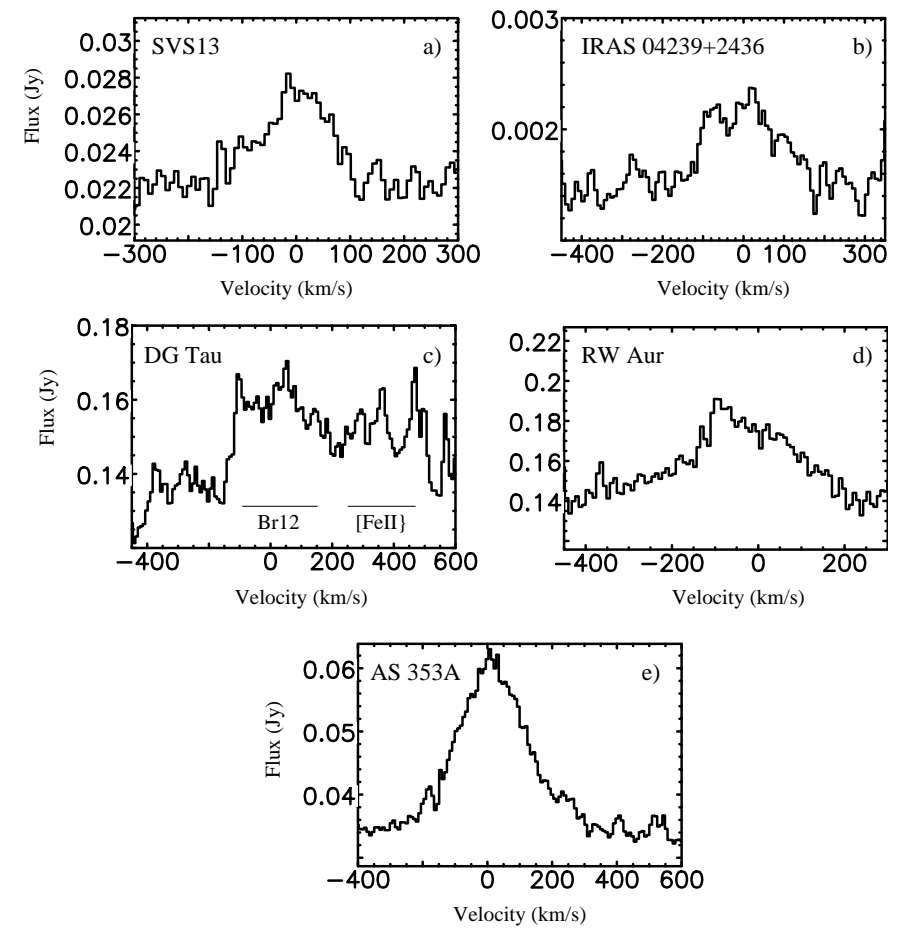

Fig. 5. Br12 spectra towards the source continuum positions in five regions (the sum of three adjacent rows is again plotted). Br12 was not detected in any of the other YSOs. Stationary [FeII] emission would be offset by $+512 \mathrm{~km} \mathrm{~s}^{-1}$ in these spectra.

$N_{\mathrm{e}} \sim 10^{4} \mathrm{~cm}^{-3}$ a ratio of 5 is expected (Hummer \& Storey 1987); this ratio is observed in dense shock regions and PDRs like Orion Peak-1 and Hubble 12 (Everett et al. 1995; Luhman \& Rieke 1996). Assuming similar excitation conditions, the $\mathrm{Br} \gamma / \mathrm{Br} 12$ ratio may therefore be used to roughly estimate the extinction to the HI region. The difference in extinction at $1.6 \mu \mathrm{m}$ and $2.2 \mu \mathrm{m}$ may be written as:

$A_{1.6}-A_{2.2} \sim 2.5 \log \left(0.2 \times I_{\mathrm{Br} \gamma} / I_{\mathrm{Br} 12}\right)$

where $I_{\mathrm{Br} \gamma} / I_{\mathrm{Br} 12}$ is the ratio of integrated $\mathrm{Br} \gamma$ and $\mathrm{Br} 12$ line fluxes (observed here and in Paper I). If $A_{2.2}=0.112 A_{\mathrm{v}}$, and $A_{1.6}=0.175 A_{\mathrm{v}}$ (Rieke \& Lebofsky 1985), then for the Class I sources where $I_{\mathrm{Br} 12} / I_{\mathrm{Br} \gamma} \sim 140$ an extinction of $A_{\mathrm{v}} \sim 60$ is predicted. This is not unreasonable if the $\mathrm{HI}$ emission lines derive from the inner regions of the accretion disk and/or the first few AU of the jet, as we argue below.

In Paper I we identified the Br $\gamma$ emission with hot gas in the inner disk and accretion flow, rather than with outflow material, because the $\operatorname{Br} \gamma$ emission was found to be spatially coincident with each source and confined to the source position (i.e. the emission was not extended along the outflow axis, unlike the $\mathrm{H}_{2}$ and [FeII]). The $\mathrm{Br} \gamma$ profiles were also found to be very broad, typically $\geq 200 \mathrm{~km} \mathrm{~s}^{-1} F W H M$, symmetric in shape, and slightly blue-shifted, typically by $10-30 \mathrm{~km} \mathrm{~s}^{-1}$. Such profiles could be due to a combination of keplerian rotation in the inner regions of a circumstellar disk (within 0.1-0.01 AU of a $1 M_{\odot}$ star) and magnetospheric accretion (Hartmann et al. 1994; Muzerrolle et al. 1998), although accretion models do tend to produce red-shifted absorption features, which were not 
observed in Paper I and are statistically rare in optical and nearIR surveys of HI emission from T Tauri stars (e.g. Reipurth et al. 1996; Folha \& Emerson 2000). In any case, the Br12 emission in the five YSOs observed here appears to have a similar origin, since the profiles in Fig. 5 are all broad and they peak at low radial velocities. From Gaussian fits to cuts made perpendicular to the dispersion axes in our $P-V$ plots, we also find that the $\mathrm{Br} 12$ peaks are coincident with the stellar continuum centroids (to an accuracy of $<1^{\prime \prime}$ ), and that the emission is not extended along the slit/outflow axes.

The one possible exception to the characteristics described above is RW Aur. Here the Br12 profile is blue-shifted and clearly asymmetric. The velocity shift and the extensive blue wing evident in Fig. 5d could be explained in terms of emission from an outflow, although we do not see clear evidence in our data that the Br12 emission is extended along the jet axis. The permitted HI Brackett lines are not usually detected in outflows on large, arcsecond ( $>100$ AU) scales. However, HI may be excited at the very base of some CTTS jets. In the optical, Takami et al. (2001) have measured spatial offsets - on AU scales - in the high-velocity wings of their $\mathrm{H} \alpha$ spectra of the CTTS RU Lupi. This suggests that the emission could, at least in part, be excited in the flow. Consequently, although the excitation conditions necessary for Brackett line emission are typically not met in the extended outflow lobes and $\mathrm{HH}$ objects, they may be met in a jet within a few AU of the central source. Higher-resolution, near-IR spectro-astrometric observations of embedded YSOs, similar to those acquired by Takami et al. (2002) for DG Tau, are urgently needed to study these regions in more detail.

Finally, we briefly consider why we do not detect Br12 (nor indeed $\mathrm{Br} \gamma$ in Paper I) towards all of the YSOs observed. Although extinction and, to a lesser extent, differing excitation conditions may play a role, the lack of $\operatorname{Br} \gamma$ and $\operatorname{Br} 12$ emission towards the majority of the Class I sources may be due to the fact that the embedded source, and therefore the line emission region, is not observed directly. If this is the case, then the continuum emission we detect in the $K$-band and, particularly, the $H$-band may be nebulosity associated with the YSO that is slightly offset from the true source position, rather than photospheric emission from the protostar. On the other hand, if HI emission is observed (as is the case for two of the most interesting Class I YSOs, SVS 13 and HH 34-IRS), then we may be confident that we are observing the source directly, and that the offsets reported above (and in Paper I) between the MHEL and FEL regions and the continuum centroids are indeed between the emission line regions and the central stars in each case.

\section{Comparison of the [Fell] data with the $\mathrm{H}_{2}$ observations in Paper I}

Having described the [FeII] and $\mathrm{H}_{2}$ observations in Sect. 4 above and in Paper I, we now compare the two data sets for each source in some detail. To aid with this comparison, we plot in Fig. $6 \mathrm{H}_{2}$ and [FeII] spectra on the same plot axes. In both cases the spectra represent the sum of three adjacent rows centred on the source continuum position, so they effectively cover the same area on each YSO. We assume that any slight

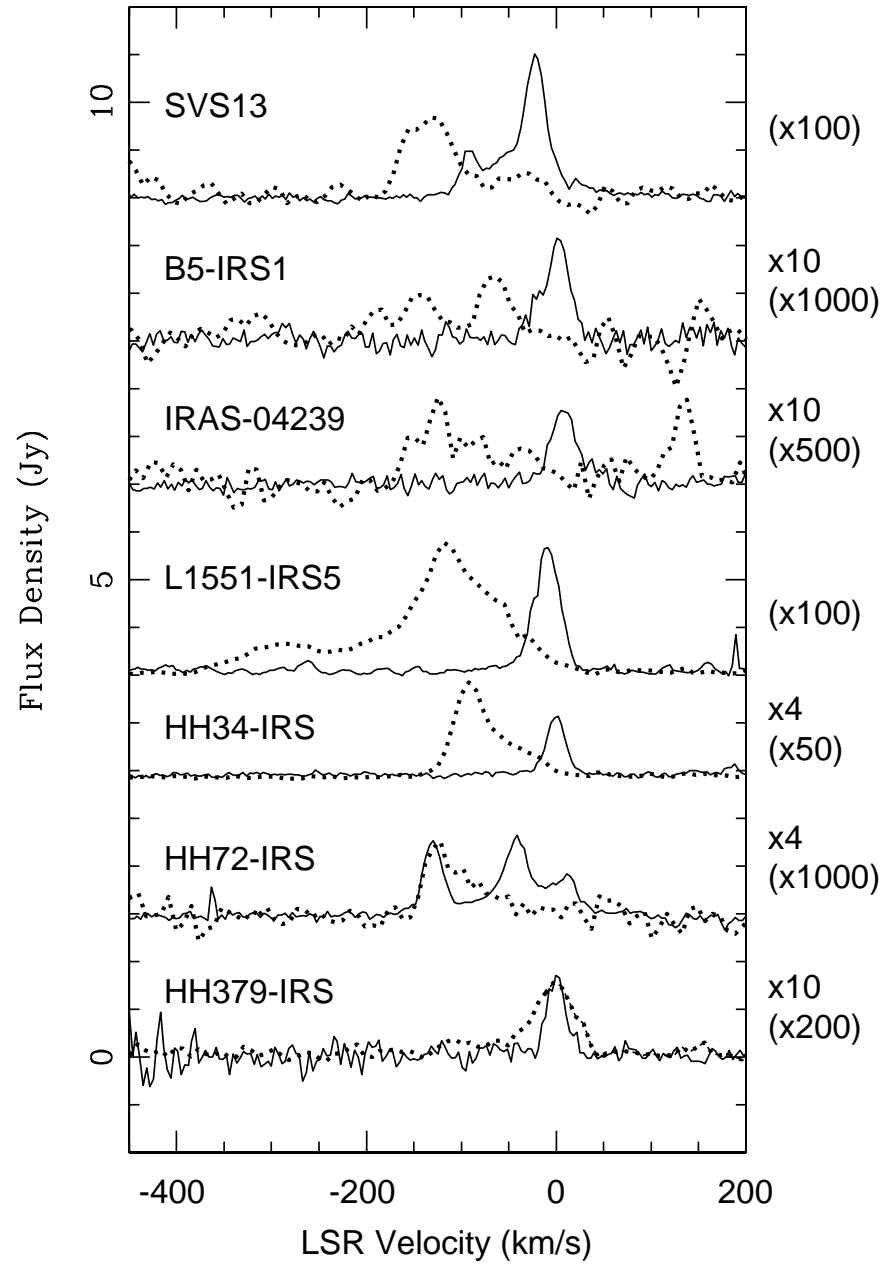

Fig. 6. A comparison of the [FeII] and $\mathrm{H}_{2}$ spectra extracted from the source-continuum position in each target (the $\mathrm{H}_{2}$ and [FeII] spectra represent the same 3-row-wide area on each source). $\mathrm{H}_{2}$ spectra are drawn with full lines; [FeII] data with thick, dotted lines. The spectra have been multiplied by the values shown to the right of each plot $\left(\mathrm{H}_{2}\right.$ multiplication factor is unbracketted; [FeII] factor is bracketted). A constant has also been added to each plot so that all data can be included in the same figure.

spatial shift in the absolute position of the slit with respect to the source, caused by a combination of orientation and the change in extinction between the $H$ and $K$-bands, is insignificant.

Line peak velocities and line widths derived from the spectra in Fig. 6 are listed in Table 2. In most cases these are measured from multi-component Gaussian fits to each profile, although for B 5-IRS1, L 1551-IRS 5 and HH 34-IRS the parameters were measured "by eye" from the spectra, since these lines are clearly non-Gaussian in shape. The errors on the individual velocities are probably dominated by systematic effects (as described in Sect. 2) rather than by errors in the fitting; errors in $V_{\text {peak }}$ and $V_{F W H M}$ are therefore of the order of $<10 \mathrm{~km} \mathrm{~s}^{-1}$.

In L 1551-IRS5 and HH 34, where complex line emission spectra were detected along the jet axes, in both $\mathrm{H}_{2}$ and [FeII], we also show (in Fig. 7) $P-V$ diagrams plotted side-by-side, and discuss these jets further in Sect. 7.2. 


\subsection{Line emission towards each outflow source}

Towards SVS 13, the [FeII] and $\mathrm{H}_{2}$ profiles in Fig. 6 are both complex and double-peaked. The $\mathrm{H}_{2}$ components peak at lower radial LSR velocities, $-20( \pm 5) \mathrm{km} \mathrm{s}^{-1}$ (LVC) and $-90( \pm 5) \mathrm{km} \mathrm{s}^{-1}$ (intermediate-velocity component, or IVC), as compared to $-35( \pm 15) \mathrm{km} \mathrm{s}^{-1}$ (LVC) and $-133( \pm 10) \mathrm{km} \mathrm{s}^{-1}$ (HVC) in [FeII]. The most significant difference in these data is the fact that, in $\mathrm{H}_{2}$, the intermediate-velocity component (IVC) is weaker than the LVC, while in [FeII] the opposite is the case - the HVC dominates. Also, overall the [FeII] profile is about twice as broad as the $\mathrm{H}_{2}$ profile. It therefore seems likely that, although both low- and intermediate/high-velocity components are observed in $\mathrm{H}_{2}$ and [FeII], the latter is nevertheless a better tracer of the highest flow velocities at the base of the jet.

$\mathrm{H}_{2}$ and [FeII] emission profiles observed towards B5-IRS 1 are also shown in Fig. 6. The $\mathrm{H}_{2}$ profile is mildly asymmetric, exhibiting a weak blue-shifted wing that extends out to about $-40 \mathrm{~km} \mathrm{~s}^{-1}$. The line profile is centred at $\sim+4( \pm 5) \mathrm{km} \mathrm{s}^{-1}$, close to the systemic velocity of $\sim 10 \mathrm{~km} \mathrm{~s}^{-1}$, though it extends over almost $100 \mathrm{~km} \mathrm{~s}^{-1}$ FWZI. The [FeII] profile may be doublepeaked, though our detection is marginal. Overall, the [FeII] emission appears to be blue-shifted with respect to the $\mathrm{H}_{2}$.

Towards IRAS $04239+2436$ only a single LVC is observed in $\mathrm{H}_{2}$; the profile peaks within a few $\mathrm{km} \mathrm{s}^{-1}$ of the systemic velocity. By comparison, [FeII] traces much higher radial velocities, in both the blue and red jet lobes (Table 2). Note, however, that for the [FeII] observations we used a different slit PA of $59^{\circ}$ (an angle of $45^{\circ}$ was used for the $\mathrm{H}_{2}$ spectroscopy in Paper I), which is better aligned with the jet axis.

Towards the $H$-band continuum position of L 1551-IRS 5 a multi-component [FeII] spectrum is observed (Fig. 6); at least two components are identified, a bright $\mathrm{HVC}$ at $\sim-120 \mathrm{~km} \mathrm{~s}^{-1}$ and an EHVC at $-285 \mathrm{~km} \mathrm{~s}^{-1}$. In $\mathrm{H}_{2}$, a single LVC is observed, peaking at approximately $-7 \mathrm{~km} \mathrm{~s}^{-1}$, though there is a blueshifted "bump" at $\sim-25 \mathrm{~km} \mathrm{~s}^{-1}$ superimposed onto this, otherwise Gaussian, line profile.

Near HH 34-IRS the [FeII] is strongly blue-shifted, while the much narrower $\mathrm{H}_{2}$ profile peaks at a relatively low velocity (Table 2). The $\mathrm{H}_{2}$ peak velocity is considerably lower towards the source position than it is along the jet, while in [FeII] the emission towards HH 34-IRS is almost as high as it is along the jet (described further below).

The [FeII] emission from HH 72-IRS peaks at $V_{\mathrm{LSR}} \sim$ $-130 \mathrm{~km} \mathrm{~s}^{-1}$; a broad red wing extends to near-zero radial velocities. By comparison, very complex $\mathrm{H}_{2}$ line emission is observed towards HH72-IRS. The $\mathrm{H}_{2}$ profile comprises at least three velocity peaks, an LVC at $+13 \mathrm{~km} \mathrm{~s}^{-1}$, an IVC at $-40 \mathrm{~km} \mathrm{~s}^{-1}$ and an HVC $-130 \mathrm{~km} \mathrm{~s}^{-1}$; these are superimposed on to a blue wing that extends to a velocity of $\sim-165 \mathrm{~km} \mathrm{~s}^{-1}$. The [FeII] peak appears to be associated only with the most blue-shifted component, the HVC.

Lastly, towards $\mathrm{HH} 379-\mathrm{IRS}$, the $\mathrm{H}_{2}$ and [FeII] profiles peak at almost the same, low, radial velocity (Table 2). This is in stark contrast to the other sources in Fig. 6; in all other sources the $[\mathrm{FeII}]$ towards the central YSO is strongly blue-shifted. These low $\mathrm{H}_{2}$ and [FeII] radial velocities in HH 379-IRS could simply be due to the orientation of the flow with respect to the line of sight (which is not well known), if the flow lies in the plane of the sky. The [FeII] may still trace the higher-velocity jet component. Notably, the [FeII] line is about twice as broad as the $\mathrm{H}_{2}$ profile, as would be expected in such a scenario (Table 2).

To summarise then, within a distance along each outflow axis of less than an arcsecond (i.e. within approximately $140 \mathrm{AU}-1500 \mathrm{AU}$ of the outflow source, depending on the distance to the target), for all Class I sources observed, the [FeII] emission is accelerated to much higher radial velocities than the $\mathrm{H}_{2}$ emission (see also Table 2).

\section{2. $\mathrm{H}_{2}$ vs [Fell] in the L $1551-I R S 5$ and HH34 jets}

Complex $\mathrm{H}_{2}$ and [FeII] line emission is also observed along the inner jet regions in L 1551-IRS 5 and HH 34. In Fig. 7 we show these $P-V$ diagrams together for ease of comparison.

In the L 1551-IRS 5 system we detect $\mathrm{H}_{2}$ line emission in both the blue jet and (weakly) in the red-shifted counterjet. The counterjet is not detected in [FeII], presumably because of increased extinction at these shorter wavelengths. In the southwestern blue lobe (negative offsets in Fig. 7) the $\mathrm{H}_{2}$ velocities are generally much lower than the [FeII] velocities. A bright $\mathrm{H}_{2}$ feature is observed at an offset of $-6^{\prime \prime}$ with a radial velocity of $-55 \mathrm{~km} \mathrm{~s}^{-1}$ which has no obvious compact [FeII] counterpart (although diffuse [FeII] is detected in this region with $V_{\mathrm{LSR}} \sim-100 \mathrm{~km} \mathrm{~s}^{-1}$ ), and double-peaked $\mathrm{H}_{2}$ is observed towards knot PHK 3 (as compared to the single, narrow [FeII] component). For PHK 3, the combined [FeII] and $\mathrm{H}_{2}$ observations can be understood in terms of a geometrical bow shock model, if the $\mathrm{H}_{2}$ is excited in the oblique bow wings, with the [FeII] produced in the high-velocity/high-excitation bow shock cap (e.g. Hartigan et al. 1987; Tedds et al. 1999).

Along the HH34-IRS jet axis (positive offsets in Fig. 7) the $\mathrm{H}_{2}$ and [FeII] emission features peak at very similar blueshifted velocities, even though the $\mathrm{H}_{2}$ and [FeII] emissions are clearly excited in different regions of the flow. $\mathrm{H}_{2}$ is observed just ahead of knot $\mathrm{L}$ and between the source and the first optically-bright $\mathrm{HH}$ knot in the jet (knot E), while the [FeII] is brightest between these two regions, at offsets of $10^{\prime \prime}-25^{\prime \prime}$. The [FeII] profiles along the jet clearly comprise a blue-shifted peak $\left(V_{\mathrm{LSR}} \sim-120 \mathrm{~km} \mathrm{~s}^{-1}\right)$ plus an extended red-wing. At higher spectral resolution these profiles would probably appear double-peaked between knots $\mathrm{E}$ and I, though the [FeII] profiles furthest from the source (towards knot $\mathrm{J}$ and $\mathrm{K}$ ) converge to a single, slightly less blue-shifted radial velocity of about $-100 \mathrm{~km} \mathrm{~s}^{-1}$. By comparison, the $\mathrm{H}_{2}$ profiles along the jet are narrower, single-peaked and centred at a radial velocity of $\sim-90 \mathrm{~km} \mathrm{~s}^{-1}$. Collectively, these [FeII] and $\mathrm{H}_{2}$ characteristics can, once again, be understood in terms of excitation in unresolved bow shocks. We identify the [FeII] emission features with the optical knots E-K (Bührke et al. 1988; Ray et al. 1996). These features are spatially resolved in the HST images of Ray et al. (1996). The double-peaked [FeII] profile at offsets of $18^{\prime \prime}-20^{\prime \prime}$ probably derives from the bow-shock shaped knot I, which in [SII] is one of the broadest and brightest knots in the inner jet region. The [FeII] line widths (and indeed the 

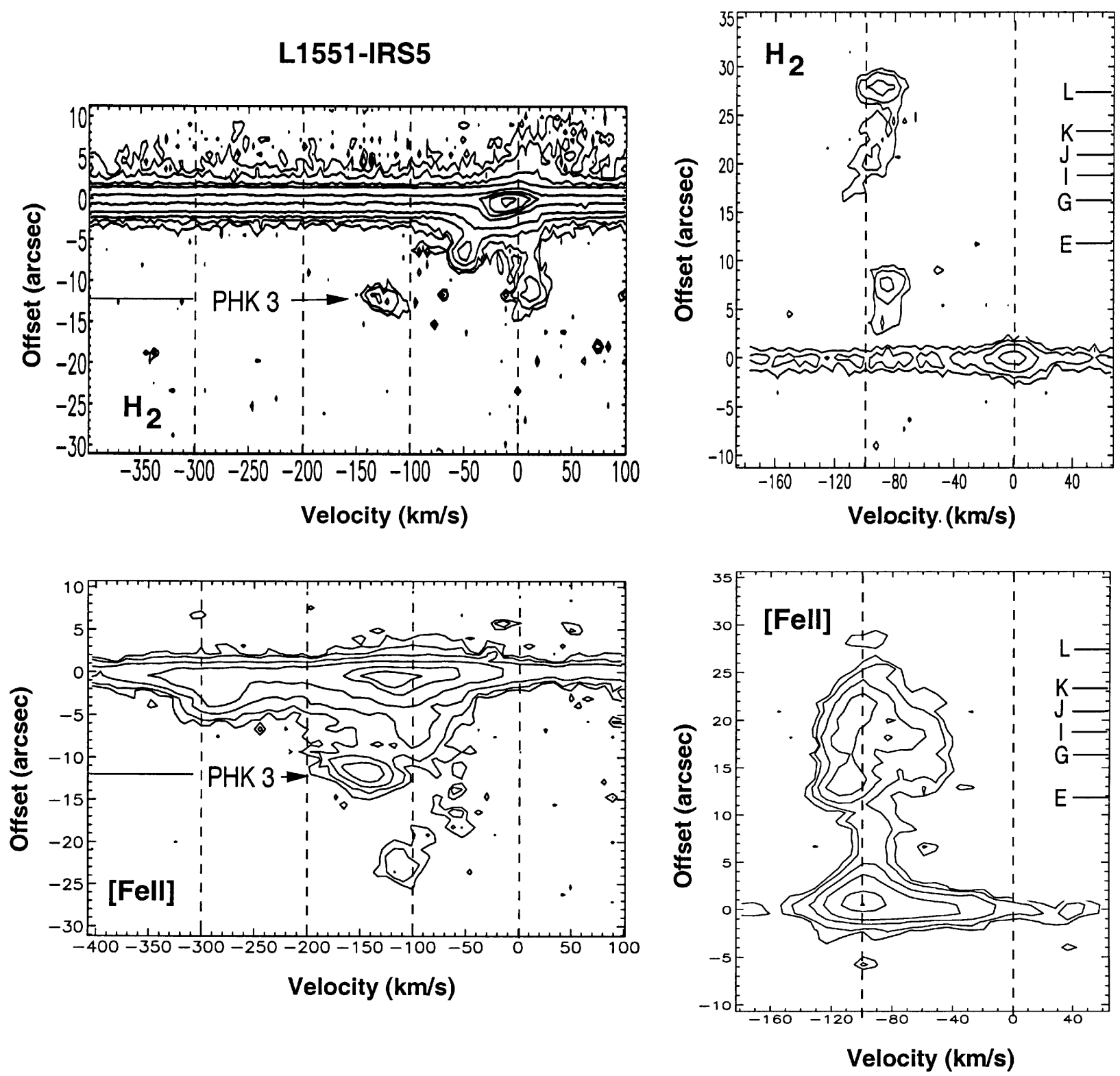

Fig. 7. A comparison of the [FeII] and $\mathrm{H}_{2} P-V$ diagrams for L 1551-IRS 5 and HH 34-IRS. In each plot the contours increase in multiples of the $1 \sigma$ blank-sky noise level: $3,5,10,20,50,75$, and 100 . The orientation of each $P-V$ plot on the sky is the same as in Figs. 1d and 1e. The systemic rest velocity for L 1551-IRS5 and HH 34-IRS is $+6 \mathrm{~km} \mathrm{~s}^{-1}$ and $+8 \mathrm{~km} \mathrm{~s}^{-1}$ respectively.

presence of [FeII] emission in the HH34 jet), suggests excitation in J-type shocks with velocities of the order of $100 \mathrm{~km} \mathrm{~s}^{-1}$. Because each bow is running into fast-moving pre-shock gas, the $\mathrm{H}_{2}$ profiles may also be strongly blue-shifted (because the gas is non-stationary) although the lines will be much narrower, since the molecular gas must be excited (rather than dissociated) in the oblique bow shock wings, where incident shock velocities will be low.

\section{FELs and MHELs - Emission from the base of an outflow}

The [FeII] observations reported in this paper have kinematic and spatial characteristics that are very similar to [SII] observations of jets from YSOs, in the outflows from the Class I YSOs and the CTTS jets. We may be certain, therefore, that [FeII] is a powerful tracer of FEL regions towards HH energy sources, particularly at high velocities and amongst the more deeply-embedded near-IR Class I sources.

We have compared in detail the [FeII] data with the $\mathrm{H}_{2}$ observations from Paper I. We find that the $\mathrm{H}_{2}$ traces a lowvelocity molecular flow component (which is notably absent from the jet driven by the only $\mathrm{T}$ Tauri star observed in Paper I, AS 353A) while the [FeII] traces intermediate and high-velocity gas (from both Class I YSOs and CTTSs).

Towards the Class I outflow sources themselves, the [FeII] profiles are usually very wide and strongly blue-shifted, to 
Table 2. Characteristics of the different velocity components seen in [FeII] and $\mathrm{H}_{2}$.

\begin{tabular}{|c|c|c|c|c|c|c|c|c|}
\hline $\begin{array}{l}\text { Source/ } \\
\text { velocity } \\
\text { Component }\end{array}$ & $\begin{array}{c}\text { [FeII }] \\
{ }^{1} V_{\text {peak }} \\
\left(\mathrm{km} \mathrm{s}^{-1}\right)\end{array}$ & $\begin{array}{c}\text { [FeII }] \\
{ }^{2} \Delta V_{F W H M} \\
\left(\mathrm{~km} \mathrm{~s}^{-1}\right)\end{array}$ & $\begin{array}{c}\text { [FeII }] \\
{ }^{3} \alpha_{[\mathrm{FeII}]} \\
\text { (degrees) }\end{array}$ & $\begin{array}{c}\mathrm{H}_{2} \\
{ }^{1} V_{\text {peak }} \\
\left(\mathrm{km} \mathrm{s}^{-1}\right)\end{array}$ & $\begin{array}{c}\mathrm{H}_{2} \\
{ }^{2} \Delta V_{F W H M} \\
\left(\mathrm{~km} \mathrm{~s}^{-1}\right)\end{array}$ & $\begin{array}{c}\mathrm{H}_{2} \\
{ }^{3} \alpha_{\mathrm{H}_{2}} \\
\text { (degrees) }\end{array}$ & $\begin{array}{c}{ }^{4} V_{\text {sys }} \\
\left(\mathrm{km} \mathrm{s}^{-1}\right)\end{array}$ & $\begin{array}{c}{ }^{5} \theta \\
\text { (degrees) }\end{array}$ \\
\hline SVS 13 & & & & & & & 8 & $40( \pm 5)$ \\
\hline LVC & -35 & $\sim 67$ & 100 & -20 & 34 & 86 & & \\
\hline IVC & & & & -89 & $\sim 36$ & 32 & & \\
\hline HVC & -133 & 70 & 42 & & & & & \\
\hline B5-IRS $1^{6}$ & & & & & & & 10 & $77( \pm 10)$ \\
\hline LVC & & & & 4 & 35 & 105 & & \\
\hline HVC & - & $\sim 46$ & - & & & & & \\
\hline IRAS $04239+2436$ & & & & & & & 8 & $60( \pm 20)$ \\
\hline LVC & & & & 7 & 35 & 174 & & \\
\hline HVC & -125 & $\sim 57$ & 24 & & & & & \\
\hline L1551-IRS5 $^{6}$ & & & & & & & 6 & $45( \pm 10)$ \\
\hline LVC & & & & -7 & 28 & 113 & & \\
\hline HVC & -120 & 111 & 64 & & & & & \\
\hline EVC & -285 & 124 & 34 & & & & & \\
\hline HH $34-$ IRS $^{6}$ & & & & & & & 8 & $68( \pm 5)$ \\
\hline LVC & & & & 1 & 16 & 81 & & \\
\hline HVC & -95 & $\sim 57$ & 23 & & & & & \\
\hline HH 72-IRS & & & & & & & 20 & $\geq 45$ \\
\hline LVC & & & & 13 & 24 & 135 & & \\
\hline IVC & & & & -40 & 39 & 49 & & \\
\hline HVC & -130 & $\sim 46$ & 24 & -129 & 19 & 10 & & \\
\hline HH 379-IRS & & & & & & & 4 & - \\
\hline LVC & 0 & 53 & - & 4 & 15 & - & & \\
\hline
\end{tabular}

Parameters for the FEL regions (from the [FeII] data) and MHEL regions $\left(\mathrm{H}_{2}\right.$ data from Paper I) extracted from the spectra in Fig. 6. The [FeII] and $\mathrm{H}_{2}$ spectra cover the same effective area on each source, though note that source distances vary, in the range $140 \mathrm{pc}$ to $1.7 \mathrm{kpc}$ (see Table 3 ). ${ }^{1}$ Velocity of the component peak. This radial velocity is not corrected for the flow inclination angle, nor has the systemic LSR velocity been subtracted.

${ }^{2} F W H M$ of the extracted profile, with the instrumental profile deconvolved (i.e. $\left.\Delta V_{F W H M}=\sqrt{(} \Delta V_{\text {observed }}^{2}-\Delta V_{\text {instrumental }}^{2}\right)$.

${ }^{3}$ Opening angle attributed to each individual flow component; $\alpha=2 \arctan \left(\Delta V_{F W H M} \times \cos \theta /\left|V_{\text {peak }}-V_{\text {sys }}\right|\right)$.

${ }^{4}$ Systemic LSR velocity of the region (see text for references).

${ }^{5}$ Inclination angle of the flow with respect to the line of sight (see Paper I for references).

${ }^{6}$ The $[\mathrm{FeII}]$ parameters for these sources were measured "by eye" from the spectra, rather than by fitting a Gaussian to the profile.

radial velocities comparable with the rest of the jet further downstream, while the $\mathrm{H}_{2}$ emission towards the source is only slightly blue-shifted, by a few tens of $\mathrm{km} \mathrm{s}^{-1}$, even though higher-velocity $\mathrm{H}_{2}$ is often detected further downstream (see e.g. HH 34 and L 1551-IRS 5 in Fig. 7). Also, the [FeII] emission peaks are spatially further offset along the jet axes than the $\mathrm{H}_{2}$ peaks (compare Fig. 4 above with the same graphs in Fig. 4 of Paper I). It therefore seems likely that the [FeII] emission is more closely tied to emission knots and shock fronts along the extended jet axes, while the $\mathrm{H}_{2}$ is predominantly excited closer to the outflow source in each system, where ambient gas densities rise sharply. The slow-moving $\mathrm{H}_{2}$ probably derives from the boundary between the jet and the stationary ambient gas.

In Table 2 we compare the line peak velocities and the line widths of the individual velocity components observed in $\mathrm{H}_{2}$ and [FeII]. From these data we crudely estimate an opening angle, $\alpha$, for each velocity component, from the velocity of the emission peak and the component width (we assume that the velocity dispersion, $\left.\Delta V=\Delta V_{F W Z I} \sim 2 \times \Delta V_{F W H M}\right)$. These data are corrected for outflow inclination angle (where known) and the systemic velocity. The estimated angles are at best only upper limits, since each line component width will be broadened by dynamical processes other than the lateral expansion of the jet (e.g. turbulence, rotation, etc.). Nevertheless, the higher-velocity jet components, seen predominantly in [FeII], are probably more highly collimated than the lower-velocity molecular components seen (in all cases) in $\mathrm{H}_{2}$, even though the $[\mathrm{FeII}]$ profiles are in all cases broader.

In Table 3 we then list integrated line intensities measured from each extracted $\mathrm{H}_{2}$ and [FeII] spectrum in Fig. 6. From these we are able to estimate a mass outflow rate and momentum for the flow components seen in $\mathrm{H}_{2}$ and [FeII] respectively. $\dot{M}_{\mathrm{H}_{2}}$ and $M V_{\mathrm{H}_{2}}$ are taken directly from Paper I. $\dot{M}_{[\mathrm{FeII}]}$ and $M V_{[\mathrm{FeII}]}$ are derived in essentially the same way. 
Table 3. Integrated line intensities.

\begin{tabular}{|c|c|c|c|c|c|c|c|c|}
\hline Source & $\begin{array}{c}{ }^{1} d \\
(\mathrm{pc})\end{array}$ & $\begin{array}{c}A_{\mathrm{v}} \\
\text { (mags) }\end{array}$ & $\begin{array}{c}{ }^{2} I_{[\mathrm{FeII}]} \\
\left(\mathrm{W} \mathrm{m}^{-2}\right)\end{array}$ & $\begin{array}{c}{ }^{2} I_{\mathrm{H}_{2} \mathrm{~S}(1)} \\
\left(\mathrm{W} \mathrm{m}^{-2}\right)\end{array}$ & $\begin{array}{c}{ }^{3} \dot{M}_{[\mathrm{FeII}]} \\
\left(M_{\odot} \mathrm{yr}^{-1}\right)\end{array}$ & $\begin{array}{c}{ }^{3} \dot{M}_{\mathrm{H}_{2}} \\
\left(M_{\odot} \mathrm{yr}^{-1}\right) \\
\end{array}$ & $\begin{array}{c}{ }^{4} M V_{[\mathrm{FeII}]} \\
\left(M_{\odot} \mathrm{km} \mathrm{s}^{-1}\right)\end{array}$ & $\begin{array}{c}{ }^{4} M V_{\mathrm{H}_{2}} \\
\left(M_{\odot} \mathrm{km} \mathrm{s}^{-1}\right)\end{array}$ \\
\hline SVS 13 & 220 & 30 & $4.2 \times 10^{-18}$ & $3.7 \times 10^{-16}$ & $8.9 \times 10^{-7}$ & $7.0 \times 10^{-7}$ & $1.9 \times 10^{-3}$ & $6.5 \times 10^{-4}$ \\
\hline B5-IRS1 & 350 & 10 & $3 \times 10^{-19}$ & $4.9 \times 10^{-17}$ & $9 \times 10^{-9}$ & $1.7 \times 10^{-8}$ & $2 \times 10^{-5}$ & $2.5 \times 10^{-5}$ \\
\hline IRAS 04239+2436 & 140 & 30 & & & & & & \\
\hline blue-jet & & & $1.0 \times 10^{-18}$ & $1.7 \times 10^{-17}$ & $2.6 \times 10^{-7}$ & $3 \times 10^{-9}$ & $2.6 \times 10^{-4}$ & $3 \times 10^{-6}$ \\
\hline red-jet & & & $4 \times 10^{-19}$ & - & $9.8 \times 10^{-8}$ & - & $1.0 \times 10^{-4}$ & - \\
\hline L1551-IRS5 & 140 & 18 & $1.1 \times 10^{-17}$ & $2.1 \times 10^{-16}$ & $1.7 \times 10^{-7}$ & $4.1 \times 10^{-8}$ & $2.1 \times 10^{-4}$ & $2.4 \times 10^{-5}$ \\
\hline HH 34-IRS & 450 & 5 & $7.2 \times 10^{-18}$ & $1.9 \times 10^{-17}$ & $7.0 \times 10^{-8}$ & $3 \times 10^{-9}$ & $2.1 \times 10^{-4}$ & $7 \times 10^{-6}$ \\
\hline HH 72-IRS & 1500 & - & $3 \times 10^{-19}$ & $9.4 \times 10^{-17}$ & $>2 \times 10^{-9}$ & $>5.8 \times 10^{-7}$ & $>3 \times 10^{-5}$ & $>3.7 \times 10^{-3}$ \\
\hline HH 379-IRS & 900 & - & $1.8 \times 10^{-18}$ & $1.0 \times 10^{-17}$ & $>2 \times 10^{-10}$ & $>7 \times 10^{-10}$ & $>1 \times 10^{-6}$ & $>3 \times 10^{-6}$ \\
\hline
\end{tabular}

${ }^{1}$ Distance to each source.

${ }^{2}$ Integrated line intensities measured across all velocity components listed in Table 2 (taken from the spectra in Fig. 6); the values for $\mathrm{H}_{2}$ are taken from Paper I. Errors on the [FeII] values are of the order of $0.1-0.3 \times 10^{-18} \mathrm{~W} \mathrm{~m}^{-2} . I_{[\mathrm{FeII}]}$ and $I_{\mathrm{H}_{2} \mathrm{~S}(1)}$ are not corrected for extinction.

${ }^{3}$ Mass outflow rate, derived using the peak velocity measured in each line (corrected for inclination angle) and the integrated line intensities in Cols. 4 and 5, corrected for the extinction, $A_{\mathrm{v}}$, in Col. 3 .

${ }^{4}$ Momentum, corrected for inclination angle and extinction.

The observed line intensity, $I_{[\mathrm{FeII}]}$, is related to the column density of $\mathrm{Fe}^{+}$ions in the upper state of the $1.64 \mu \mathrm{m}$ transition, $N_{\mathrm{u}}$, by $I_{[\mathrm{FeII}]}=N_{\mathrm{u}} h v_{\mathrm{ul}} A_{\mathrm{ul}} /(4 \pi)$, where $I_{[\text {FeII }]}$ is measured in $\mathrm{W} \mathrm{m} \mathrm{m}^{-2} \operatorname{ster}^{-1}, A_{\mathrm{ul}}$ is the radiative decay rate of the $1.64 \mu \mathrm{m}$ transition $\left(A_{\mathrm{ul}}=0.00465 \mathrm{~s}^{-1}\right.$; Nussbaumer \& Storey 1988) and $v_{\mathrm{ul}}$ is the frequency of the transition. The total column density of hydrogen atoms and ions, $N_{\mathrm{H}}$, is then given by $N_{\mathrm{H}}=N_{\mathrm{u}} \times(\mathrm{H} / \mathrm{Fe}) /\left(f_{\mathrm{u}} f_{\mathrm{Fe}+}\right)$, where $f_{\mathrm{u}}$ and $f_{\mathrm{Fe}+}$ are the fraction of $\mathrm{Fe}^{+}$ions in the upper state and the fraction of $\mathrm{Fe}$ that is singly ionised. $(\mathrm{Fe} / \mathrm{H})$ is the $\mathrm{Fe}-$ to-H abundance ratio; we adopt a solar ratio of $2.14 \times 10^{4}$ (Grevesse \& Ander 1989). Hamann et al. (1994) have calculated values of $f_{\mathrm{u}}$ over a range of electron temperatures and densities; they find that $f_{\mathrm{u}}$ is sensitive only to $n_{\mathrm{e}}$. For $n_{\mathrm{e}} \sim 10^{4} \mathrm{~cm}^{-3}$ (close to the critical density for excitation of the observed transition) a value of $f_{\mathrm{u}}=0.01$ is predicted, which we use here. Hamann (1994) also predict $f_{\mathrm{Fe}+} \sim 0.68$ under similar conditions.

The mass outflow rates and momenta in Table 3 are calculated for the areas encompassed by the 3-row-wide extracted spectra displayed in Fig. 6. In the $\mathrm{H}_{2}$ and [FeII] observations this angular area is the same, though because of the different distances to each region the actual area differs from source to source. In calculating $\dot{M}$ we assume that the emission is extended along the jet axis, i.e. $\dot{M}=M / \tau$, where $\tau$ $\left(\sim L /\left[V_{\text {peak }} \tan \theta\right]\right)$ is a dynamical time scale for the observed section of the jet. If the emission is unresolved along the jet, $\tau$ will be overestimated and $\dot{M}$ will be underestimated, although because we have already established that the emission is offset and/or extended along the axis in each system (in $\mathrm{H}_{2}$ and [FeII]), this is probably not a dominant source of uncertainty.

The largest sources of error in $\dot{M}$ and $M V$ in Table 3 are probably; (1) the value of $A_{\mathrm{v}}$ used to correct the observed intensities, $I_{[\mathrm{FeII}]}$ and $I_{\mathrm{H}_{2}}$, for extinction, (2) the inclination angle of the flow, and (3) the velocity adopted for the [FeII] and $\mathrm{H}_{2}$ flow components. The values of $A_{\mathrm{v}}$ used (references are listed in Paper I) are estimated usually from molecular column densities measured from (sub)mm observations made at low angular resolution, or optical line ratios. Clearly, because neither the submm nor the optical emission derives from the same region as the near-IR emission very near to each source, the values of $A_{\mathrm{v}}$ used in our analysis will be uncertain, and probably underestimated (because of beam dilution in the submm, or because the optical emission is detected only from lowerextinction regions further out). Low-resolution $H$-band spectroscopy would be useful to measure the extinction from the ratio of the [FeII] lines at $1.644 \mu \mathrm{m}$ and $1.257 \mu \mathrm{m}$ (e.g. Reipurth et al. 2000). Then the extinction towards the [FeII] region probably the largest source of error in Table 3 - would be measured directly. In a few sources $A_{\mathrm{v}}$ is not know at all; for $\mathrm{HH} 72-$ IRS and HH 379-IRS we do not correct the parameters in Table 3 for extinction, so these are listed as lower limits. An $A_{\mathrm{v}}$ of $\sim 25-40$ would result in an increase of the order of $10^{2}-10^{3}$ in $\dot{M}_{[\mathrm{FeII}]}$ and $M V_{\text {[FeII] }}$, and $10-40$ in $\dot{M}_{\mathrm{H}_{2}}$ and $M V_{\mathrm{H}_{2}}$. (Note, however, that a very high value of $A_{v}$ would probably render the [FeII] emission unobservable!)

The inclination angle of the jet in most cases is known to within $\sim 10 \%$, so this is probably a secondary source of uncertainty. The choice of flow velocity used to calculate $\dot{M}$ and $M V$ is, however, somewhat arbitrary. We use the radial velocity of the emission peak, $V_{\text {peak }}$, since we assume that the broad line widths observed (particularly in $[\mathrm{FeII}]$ ) are largely due to lateral expansion of the flow, turbulence in a shear layer between the jet and the stationary ambient medium, and thermal motions in the post-shock gas. An uncertainty in $V_{\text {peak }}$ of $10-50 \mathrm{~km} \mathrm{~s}^{-1}$ could nevertheless result in an additional error in $\dot{M}$ and $M V$ of a factor of 2-3. Overall then, we estimate an approximate error of the order of a factor of 10-100 on the mass loss rates and momenta listed in Table 3.

Even given the uncertainties listed above, the massloss rates and momenta in Table 3 are fairly typical of $\mathrm{HH}$ jets from low-mass YSOs, where e.g. the average mass loss rates vary from $1 \times 10^{-6}$ to $5 \times 10^{-8} M_{\odot} \mathrm{yr}^{-1}$ 
(Bacciotti \& Eislöffel 1999). In HH 34, for example, which is the most well defined jet in [SII], [FeII] and $\mathrm{H}_{2}$ emission, a mass-loss rate of $2-4 \times 10^{-7} M_{\odot} \mathrm{yr}^{-1}$ has been measured from optical emission-line studies (Heathcote \& Reipurth 1992; Bacciotti \& Eislöffel 1999); in [FeII] we derive a mass loss rate at the base of the jet of $7 \times 10^{-8} M_{\odot} \mathrm{yr}^{-1}$, which is only a factor of 3-5 lower. And in L 1551-IRS5, a momentum of $<7 \times 10^{-4} \quad M_{\odot} \mathrm{km} \mathrm{s}^{-1}$ has been predicted from recent HST observations (Fridlund \& Liseau 1998); in [FeII] we estimate $M V_{\text {[FeII }]} \sim 2 \times 10^{-4} M_{\odot} \mathrm{km} \mathrm{s}^{-1}$. The mass loss rates and momenta in Table 3 are therefore not unreasonable.

It is also worth noting that, although the absolute errors on $\dot{M}$ and $M V$ for the $\mathrm{H}_{2}$ and [FeII] flow components may be large, the relative errors between these two parameters will be much smaller, because the same extinction is used for each data set. Consequently, if the [FeII] traces a collimated jet component, then the above analysis suggests that the momentum in this jet is equal to or greater than the momentum in the more poorly-collimated, molecular outflow which we trace in $\mathrm{H}_{2}$ in each system. Moreover, given the higher velocities associated with the [FeII] jet component, the momentum supply rate, $\dot{M} V$, of each jet should be sufficient to drive the $\mathrm{H}_{2}$ flow. In other words, the [FeII] jet will supply enough momentum per unit time to entrain and accelerate the molecular flow seen in $\mathrm{H}_{2}$. There is even some evidence that this process is more efficient in the more deeply embedded outflows, like SVS 13 and B5-IRS1 (which have no optical jet) than it is in the less-embedded flows, like L 1551-IRS 5 and HH 34IRS (both YSOs have well-known optical jets observed close to the central engine), since in SVS 13 and B5-IRS1 the momentum in the [FeII] and $\mathrm{H}_{2}$ components are roughly equal, while in L 1551-IRS 5 and HH $34 M V_{\text {[FeII] }}$ is an order of magnitude greater than $M V_{\left[\mathrm{H}_{2}\right]}$.

Finally, we reiterate that there is also some evidence to suggest that the $\mathrm{H}_{2}$ component is more poorly collimated than the [FeII] jet, as one would expect if the former is entrained in a boundary layer between the jet and the ambient medium. It seems likely, therefore, that entrainment of molecular material is present in YSO jets even within a few hundred AU of the central driving source. Higher-resolution spectroscopy across the width of a few MHEL flows are clearly needed to investigate this possibility further. Moreover, X-wind and disk-wind models should also now strive to predict FEL and MHEL characteristics within the first $1000 \mathrm{AU}$ of the central outflow source, since observational data at high spatial and spectral resolution are now forthcoming.

\section{Conclusions}

[FeII] long-slit echelle spectroscopy of seven Class I YSOs and three CTTSs is presented. We detect emission towards the Hband continuum positions of all seven Class I sources (Fig. 6) and along the extended jet lobes of three of these. [FeII] emission is also detected in the HH jets of the three CTTSs; in RW Aur and DG Tau this emission is traced to within a few arcseconds of the source.
From a comparison of the [FeII] observations with published [SII] observations and the $\mathrm{H}_{2}$ observations in a companion paper (Paper I) we arrive at the following conclusions:

1. The $[\mathrm{FeII}]$ emission is typically blue-shifted to much higher velocities than the $\mathrm{H}_{2}$ emission in the FEL/MHEL region in each Class I YSO. Where low and high-velocity components are observed in $\mathrm{H}_{2}$, the [FeII] is usually brightest or even confined to the $\mathrm{HVC}$, while the $\mathrm{H}_{2}$ emission is strongest in the LVC.

2. The [FeII] emission peak is usually offset further along the jet axis (from the source continuum centroid) than the $\mathrm{H}_{2}$ emission.

3. The $[\mathrm{FeII}]$ peak velocities, range of velocities observed and the distribution in [FeII] emission along each jet axis closely follows that seen in [SII], in the Class I YSOs where the latter is observed (e.g. HH 34 and L 1551-IRS5) and in all three CTTS jets.

We suggest that the [FeII] emission derives from the base of a collimated, high-velocity jet which entrains ambient molecular gas within a few hundred AU of each $\mathrm{HH}$ energy source. The entrained gas is observed in $\mathrm{H}_{2}$ emission. Our analysis indicates that the $[\mathrm{FeII}]$ jet may well have sufficient power to drive the $\mathrm{H}_{2}$ flow.

Lastly, Br12 emission was also detected towards the CTTSs and towards two of the Class I YSOs. These HI emission profiles are very broad, though they are single-peaked and relatively symmetric in shape. The profiles peak at low blue-shifted velocities. The emission is also confined to the source position, i.e. we see no distinct offset of the emission peak along the jet axis, and we do not detect Br12 from the extended jet regions. We therefore associate the emission with the same highexcitation regions observed in $\mathrm{Br} \gamma$ in Paper I, namely the inner regions of the accretion disk, magnetospheric accretion flows, and/or the first few $\mathrm{AU}$ of the jet.

Acknowledgements. We thank Watson Varricatt for his assistance at the telescope. The UKIRT is operated by the Joint Astronomy Centre on behalf of the U.K. Particle Physics and Astronomy Research Council. The Starlink Software Collection is managed and distributed by the Starlink Project which is funded by PPARC.

\section{References}

Anglada, G., Rodríguez, L. F., \& Torrelles, J. M. 2000, ApJ, 542, L123 Bacciotti, F., \& Eislöffel, J. 1999, A\&A, 342, 717

Bacciotti, F., Hirth, G. A., \& Natta, A. 1996, A\&A, 310, 309

Bacciotti, F., Mundt, R., Ray, T. P., et al. 2000, ApJ, 537, L49

Bacciotti, F., Ray, T. P., Mundt, R., Eisloeffel, J., \& Solf, J. 2002, ApJ, 576,222

Bachiller, R., \& Cernicharo, J. 1990, A\&A, 239, 276

Bachiller, R., Gueth, F., Guilloteau, S., Tafalla, M., \& Dutrey, A. 2000, A\&A, 362, L33

Bally, J., Devine, D., \& Alten, V. 1996, ApJ, 473, 921

Bührke, T., Mundt, R., \& Ray, T. P. 1988, A\&A, 200, 99

Carr, J. S. 1993, ApJ, 406, 553

Chernin, L. M., \& Masson, C. R. 1995, ApJ, 443, 181

Chrysostomou, A., Hobson, J., Smith, M. D., Davis, C. J., \& Berndsen, A. 2000, MNRAS, 314, 229 
Curiel, S., Raga, A., Raymond, J., Noriega-Crespo, A., \& Cantó, J. 1997, AJ, 114, 2736

Davis, C. J., Eislöffel, J., \& Smith, M. D. 1996, ApJ, 463, 246

Davis, C. J., Mundt, R., Ray, T. P., \& Eislöffel, J. 1995, AJ, 110, 766

Davis, C. J., Ray, T. P., Desroches, L., \& Aspin, C. 2001, MNRAS, 326, 524 (Paper I)

Davis, C. J., Ray, T. P., Eislöffel, J., \& Corcoran, D. 1997, A\&A, 324, 263

Davis, C. J., Stern, L., Ray, T. P., \& Chrysostomou, A. 2002, A\&A, 382,1021

Devine, D., Bally, J., Reipurth, B., \& Heathcote, S. 1997a, AJ, 114, 2095

Devine, D., Reipurth, B., \& Bally, J. 1997b, in Low-mass star formation - from infall to outflow, poster Proc., ed. F. Malbet, \& A. Castets, IAU Symp., 182, 91

Dobashi, K., Bernard, J.-P., Yonekura, Y., \& Fukui, Y. 1994, ApJS, 95, 419

Dougados, C., Cabrit, S., Lavalley, C., \& Ménard, F. 2000, A\&A, 357, L61

Edwards, S., \& Snell, R. L. 1982, ApJ, 261, 151

Eislöffel, J., Günther, E., Hessman, F. V., et al. 1991, ApJ, 383, L19

Eislöffel, J., \& Mundt, R. 1992, A\&A, 263, 292

Eislöffel, J., \& Mundt, R. 1997, AJ, 114, 280

Eislöffel, J., Solf, J., \& Böhm, K. H. 1990, A\&A, 237, 369

Everett, M. E., De Poy, D. L., \& Pogge, R. W. 1995, AJ, 110, 1295

Folha, D. F. M., \& Emerson, J. P. 2000, A\&A, 365, 90

Fridlund, C. V. M., \& Liseau, R. 1998, ApJ, 499, L75

Gahm, G. F., Petrov, P. P., Duemmler, R., Gameiro, J. F., \& Lago, M. T. V. T. 1999, A\&A, 352, L95

Garden, R. P., Russell, A. P. G., \& Burton, M. G. 1990, ApJ, 354, 232

Gredel, R. 1996, A\&A, 305, 582

Greene, T. P., \& Lada, C. J. 1996, ApJ, 461, 345

Grevesse, N., \& Anders, E. 1989, in Cosmic Abundances of Matter, ed. C. I. Waddington (New York: AIP), AIOP Conf. Proc., 183, 1

Hamann, F. 1994, ApJS, 93, 485

Hamann, F., Simon, M., Carr, J. S., \& Prato, L. 1994, ApJ, 436, 292

Hartigan, P., Morse, J., Palunas, P., Bally, J., \& Devine, D. 2000, ApJ, 119,1872

Hartigan, P., Mundt, R., \& Stocke, J. 1986, AJ, 91, 1357

Hartigan, P., Raymond, J. C., \& Hartmann, L. 1987, ApJ, 316, 323

Hartmann, L., Hewett, R., \& Calvet, S. 1994, ApJ, 426, 669

Heathcote, S., \& Reipurth, B. 1992, AJ, 104, 2193

Hirth, G., Mundt, R., \& Solf, J. 1997, A\&AS, 126, 437

Hirth, G., Mundt, R., Solf, J., \& Ray, T. P. 1994, ApJ, 427, L99

Hollenbach, D., \& McKee, C. F. 1989, ApJ, 342, 306

Hummer, D. G., \& Storey, P. J. 1987, MNRAS, 224, 801

Itoh, Y., Kaifu, N., Hayashi, M., et al. 2000, PASJ, 52, 81
Johansson, S. 1978, Phys. Scr., 18, 217

Khanzadyan, T., Smith, M. D., Davis, C. J., et al. 2002, MNRAS, in press

Kitamura, Y., Kawabe, R., \& Saito, M. 1996, ApJ, 457, 277

Lavalley, C., Cabrit, S., Dougados, C., Ferruit, P., \& Bacon, R. 1997, A\&A, 327, 671

Luhman, K. L., \& Rieke, G. H. 1996, ApJ, 461, 298

Maihara, T., Iwamuro, F., Yamashita, T., et al. 1993, PASP, 105, 940

Moriarty-Schieven, G., \& Snell, R. L. 1988, ApJ, 332, 364

Mountain, C. M., Robertson, D., Lee, T. J., \& Wade, R. 1990, in Instrumentation in Astronomy, SPIE 1235, VII, 25

Mundt, R., Brugel, E. W., \& Bührke, T. 1987, ApJ, 319, 275

Mundt, R., \& Eislöffel, J. 1998, AJ, 116, 860

Mundt, R., \& Fried, J. 1983, ApJ, 274, L83

Mundt, R., Ray, T. P., \& Raga, A. C. 1991, A\&A, 252, 740

Mundt, R., Stocke, J., \& Stockman, H. S. 1983, ApJ, 265, L71

Muzerolle, J., Calvet, N., \& Hartmann, L. 1998, ApJ, 116, 2965

Nussbaumer, H., \& Storey, P. J. 1988, A\&A, 193, 327

Pyo, T.-S., Hayashi, M., Kobayashi, N., et al. 2002, ApJ, 570, 724

Ray, T. P., Mundt, R., Dyson, J. E., Falle, S. A. E. G., \& Raga, A. C. 1996, ApJ, 468, L103

Rieke, G. H., \& Lebovsky, M. J. 1985, ApJ, 288, 618

Reipurth, B., Bally, J., \& Devine, D. 1997, AJ, 114, 2708

Reipurth, B., \& Graham, J. A. 1988, A\&A, 202, 219

Reipurth, B., Pedrosa, A., \& Lago, M. T. V. T. 1996, A\&AS, 120, 229

Reipurth, B., Yu, K. C., Heathcote, S., Bally, J., \& Rodríguez, L. 2000, AJ, 120, 449

Schwartz, R. D., Gee, G., \& Huang, Y.-L. 1988, ApJ, 327, 350

Shu, F. H., Ruden, S. P., Lada, C. J., \& Lizano, S. 1991, ApJ, 370, L31

Shu, F. H., Najita, J., Ostriker, E. C., \& Shang, H. 1995, ApJ, 455, L155

Shu, F. H., Najita, J., Shang, H., \& Li, Z. Y. 2000, in Protostars and Planets IV, ed. V. Mannings, A. P. Boss, \& S. S. Russell (Tucson: Univ. Arizona Press), 789

Shull, J. M., \& Hollenbach, D. J. 1978, ApJ, 220, 525

Solf, J., Böhm, K. H., \& Raga, A. 1986, ApJ, 305, 795

Stapelfeldt, K. R., Scoville, N. Z., Beichman, C. A., Hester, J., \& Gautier, T. N. 1991, ApJ, 371, 226

Stocke, J. T., Hartigan, P., Strom, S. E., et al. 1988, ApJS, 68, 229

Takami, M., Chrysostomou, A., Bailey, J., et al. 2002, ApJ, 568, L53

Takami, M., Bailey, J., Gledhill, T. M., Chrysostomou, A., \& Hough, J. H. 2001, MNRAS, 323, 177

Ungerechts, H., \& Thaddeus, P. 1987, ApJS, 63, 645

Woitas, J., Ray, T. P., Bacciotti, F., Davis, C. J., \& Eislöffel, J. 2002, ApJ, in press

Yu, K. C., Billawala, Y., \& Bally, J. 1999, ApJ, 118, 2940 